\title{
COHEN FACTORIZATIONS: WEAK FUNCTORIALITY AND APPLICATIONS
}

\author{
SAEED NASSEH AND SEAN SATHER-WAGSTAFF \\ To Hans-Bjørn Foxby on the occasion of his sixty-fifth birthday
}

\begin{abstract}
We investigate Cohen factorizations of local ring homomorphisms from three perspectives. First, we prove a "weak functoriality" result for Cohen factorizations: certain morphisms of local ring homomorphisms induce morphisms of Cohen factorizations. Second, we use Cohen factorizations to study the properties of local ring homomorphisms (Gorenstein, Cohen-Macaulay, etc.) in certain commutative diagrams. Third, we use Cohen factorizations to investigate the structure of quasi-deformations of local rings, with an eye on the question of the behavior of CI-dimension in short exact sequences.
\end{abstract}

\section{INTRODUCTION}

Convention. Throughout this paper, the term "ring" is short for "commutative noetherian ring with identity". A local ring is "complete" when it is complete with respect to its maximal ideal. Let $R, S$, and $T$ be rings.

Cohen factorizations were introduced in [9] as tools to study local ring homomorphisms. (See Section 2 for definitions and background material.) The utility of these factorizations can be seen in their many applications; see, e.g., 3, 4, 6, 7, 8, 13, 22, 28. The main point of this construction is that it allows one to study a local ring homomorphism by replacing it with a surjective one; thus, one can assume that the target is finitely generated over the source, so one can apply finite homological algebra techniques.

In Section 3 of this paper, we investigate functorial properties of Cohen factorizations. The main result of this section is the following; its proof is in 3.4. Example 3.5 shows that the separability assumptions (2) are necessary.

Theorem A. Consider a commutative diagram of local ring homomorphisms

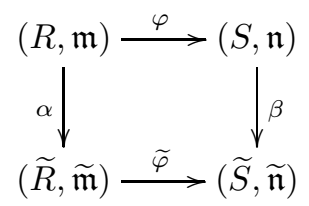

with the following properties:

2010 Mathematics Subject Classification. 13B40, 13D02, 13D05, $13 \mathrm{D} 07$.

Key words and phrases. CI-dimension, CM-dimension, Cohen factorizations, Cohen-Macaulay homomorphisms, flat dimension, G-dimension, Gorenstein homomorphisms, quasi-CohenMacaulay hommorphisms, quasi-Gorenstein homomorphisms.

Sather-Wagstaff was supported in part by North Dakota EPSCoR, National Science Foundation Grant EPS-0814442, and a grant from the NSA. 
(1) $\alpha$ and $\varphi$ have regular factorizations $R \stackrel{\dot{\alpha}}{\rightarrow} R^{\prime \prime} \stackrel{\alpha^{\prime}}{\rightarrow} \widetilde{R}$ and $R \stackrel{\dot{\varphi}}{\rightarrow} R^{\prime} \stackrel{\varphi^{\prime}}{\longrightarrow} S$, e.g., $\widetilde{R}$ and $S$ are complete,

(2) $\widetilde{S}$ is complete, and the field extensions $R / \mathfrak{m} \rightarrow \widetilde{R} / \widetilde{\mathfrak{m}} \rightarrow \widetilde{S} / \widetilde{\mathfrak{n}}$ are separable.

Let $S \stackrel{\dot{\beta}}{\rightarrow} S^{\prime} \stackrel{\beta^{\prime}}{\longrightarrow} \widetilde{S}$ and $\widetilde{R} \stackrel{\dot{\varphi}}{\rightarrow} \widetilde{R}^{\prime} \stackrel{\widetilde{\varphi}^{\prime}}{\longrightarrow} \widetilde{S}$ be Cohen factorizations of $\beta$ and $\widetilde{\varphi}$. Then there is a commutative diagram of local ring homomorphisms

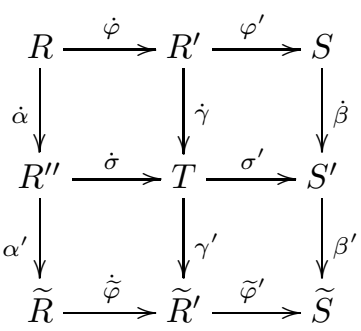

such that the diagrams $R^{\prime} \stackrel{\dot{\dot{q}}}{\rightarrow} T \stackrel{\gamma^{\prime}}{\rightarrow} \widetilde{R}^{\prime}$ and $R^{\prime \prime} \stackrel{\dot{\sigma}}{\rightarrow} T \stackrel{\sigma^{\prime}}{\rightarrow} S^{\prime}$ are minimal Cohen factorizations.

We think of this as a result about functoriality of regular (e.g., Cohen) factorizations as follows. The diagram (A.1) is a morphism in the category of local ring homomorphisms. Our result provides the following commutative diagram of local ring homomorphisms where $\gamma=\gamma^{\prime} \dot{\gamma}$

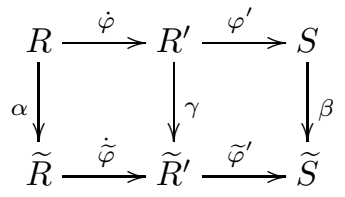

which is a morphism in the category of regular factorizations. Of course, the operation that maps a local ring homomorphism to a regular (or Cohen) factorization is not well-defined; hence our terminology "weak functoriality".

Given a diagram (A.1) where $\alpha$ and $\beta$ are "nice", the maps $\varphi$ and $\widetilde{\varphi}$ are intimately related. This maxim is the subject of Section 4, which culminates in the proof of the next result. It is one of the applications of Cohen factorizations mentioned in the title; see 4.9 for the proof.

Theorem B. Consider a commutative diagram of local ring homomorphisms

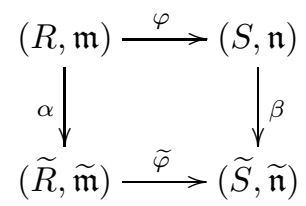

such that $\alpha$ is weakly Cohen, $\beta$ is weakly regular, and the induced map $\widetilde{R} / \widetilde{\mathfrak{m}} \rightarrow \widetilde{S} / \widetilde{\mathfrak{n}}$ is separable. Let $P$ be one of the following conditions: Gorenstein, quasi-Gorenstein, complete intersection, Cohen-Macaulay, quasi-Cohen-Macaulay. Then $\varphi$ is $P$ if and only if $\widetilde{\varphi}$ is $P$.

Section 4 also contains, among other things, a base change result for CI-dimension and CM-dimension (Proposition 4.7) which may be of independent interest. 
The paper concludes with Section 5. which is devoted almost entirely to the proof of the next result; see 5.2 It is another application of Cohen factorizations. Also, it is related to the short exact sequence question for CI-dimension, as we describe in Remark 5.1 .

Theorem C. For $i=1,2$ let $(R, \mathfrak{m}, k) \stackrel{\varphi_{i}}{\longrightarrow}\left(R_{i}, \mathfrak{m}_{i}, k_{i}\right) \stackrel{\tau_{i}}{\longleftarrow}\left(Q_{i}, \mathfrak{n}_{i}, k_{i}\right)$ be a quasideformation such that the field extension $k \rightarrow k_{i}$ is separable.

(a) Then there exists a commutative diagram of local ring homomorphisms

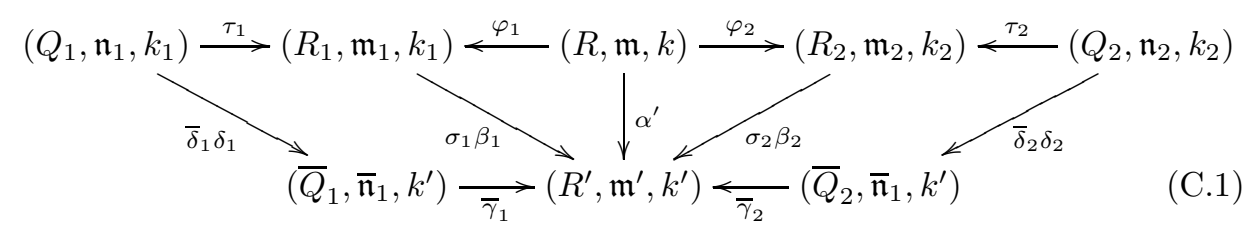

such that $\alpha^{\prime}$ is flat, each map $\bar{\delta}_{i} \delta_{i}$ is weakly Cohen, and each map $\bar{\gamma}_{i}$ is surjective. (See the proof for an explanation of the labels $\delta_{i}, \sigma_{i}$, etc.)

(b) Assume that each map $\varphi_{i}$ is weakly Cohen. Then the maps in diagram (C.1) satisfy the following properties for $i=1,2$ :

(b1) The maps $R_{i} \rightarrow R^{\prime}$ are weakly Cohen.

(b2) The diagram $R \rightarrow R^{\prime} \nleftarrow \bar{Q}_{i}$ is a quasi-deformation and each parallelogram diagram is a pushout.

(b3) Given a $R$-module $M$, if $\operatorname{pd}_{Q_{i}}\left(M \otimes_{R} R_{i}\right)<\infty$, then $\operatorname{pd}_{\bar{Q}_{i}}\left(M \otimes_{R} R^{\prime}\right)<\infty$.

As the list of references indicates, this work is built on ideas pioneered by HansBjørn Foxby, his collaborators, and his students. We are deeply grateful to him for his many contributions to this field through his research and his support.

\section{BACKGROUND}

This section lists important foundational material for use in the rest of the paper.

\section{Factorizations.}

Definition 2.1. Let $\varphi:(R, \mathfrak{m}) \rightarrow(S, \mathfrak{n})$ be a local ring homomorphism.

(a) The embedding dimension of $\varphi$ is $\operatorname{edim}(\varphi)=\operatorname{edim}(S / \mathfrak{m} S)$.

(b) The map $\varphi$ is weakly regular if it is flat and the closed fiber $S / \mathfrak{m} S$ is regular.

(c) The map $\varphi$ is a weakly Cohen if it is weakly regular, and the induced field extension $R / \mathfrak{m} \rightarrow S / \mathfrak{n}$ is separable, e.g., if $\operatorname{char}(R / \mathfrak{m})=0$ or $R / \mathfrak{m}$ is perfect of positive characteristic.

(d) The map $\varphi$ is Cohen if it is weakly Cohen such that $\mathfrak{n}=\mathfrak{m} S$, that is, it is weakly Cohen such that the closed fiber is a field.

Fact 2.2. Let $R \stackrel{\varphi}{\rightarrow} S \stackrel{\psi}{\rightarrow} T$ be weakly regular local ring homomorphisms. Then the composition $\psi \varphi$ is weakly regular such that $\operatorname{edim}(\psi \varphi)=\operatorname{edim}(\psi)+\operatorname{edim}(\varphi)$; see [22, 5.9].

The central objects of study for this paper, defined next, are from the work of Avramov, Foxby, and B. Herzog [9].

Definition 2.3. Let $\varphi:(R, \mathfrak{m}) \rightarrow(S, \mathfrak{n})$ be a local ring homomorphism.

(a) A regular factorization of $\varphi$ is a diagram of local homomorphisms $R \stackrel{\dot{\varphi}}{\rightarrow} R^{\prime} \stackrel{\varphi^{\prime}}{\rightarrow} S$ such that $\varphi=\varphi^{\prime} \dot{\varphi}$, the map $\dot{\varphi}$ is weakly regular, and $\varphi^{\prime}$ is surjective. 
(b) A Cohen factorization of $\varphi$ is a regular factorization $R \stackrel{\dot{\varphi}}{\rightarrow} R^{\prime} \stackrel{\varphi^{\prime}}{\rightarrow} S$ of $\varphi$ such that $R^{\prime}$ is complete.

(c) A comparison of one Cohen factorization $R \stackrel{\dot{\varphi}}{\rightarrow} R^{\prime} \stackrel{\varphi^{\prime}}{\rightarrow} S$ of $\varphi$ to another one $R \stackrel{\ddot{\varphi}}{\rightarrow} R^{\prime \prime} \stackrel{\varphi^{\prime \prime}}{\longrightarrow} S$ is a local homomorphism $v: R^{\prime} \rightarrow R^{\prime \prime}$ making the following diagram commute:

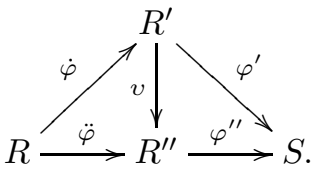

(d) The semicompletion of $\varphi$, denoted $\grave{\varphi}: R \rightarrow \widehat{S}$, is the composition of $\varphi$ with the natural map $S \rightarrow \widehat{S}$.

Remark 2.4. Let $R \stackrel{\dot{\varphi}}{\rightarrow} R^{\prime} \stackrel{\varphi^{\prime}}{\longrightarrow} S$ be a regular factorization of a local ring homomorphism $\varphi: R \rightarrow S$. The surjective homomorphism $\varphi^{\prime}: R^{\prime} \rightarrow S$ induces a surjective homomorphism $R^{\prime} / \mathfrak{m} R^{\prime} \rightarrow S / \mathfrak{m} S$ which implies that $\operatorname{edim}(\dot{\varphi}) \geqslant \operatorname{edim}(\varphi)$. Since $\dot{\varphi}$ is weakly regular, we conclude that

$$
\operatorname{dim}\left(R^{\prime}\right)-\operatorname{dim}(R)=\operatorname{edim}(\dot{\varphi}) \geqslant \operatorname{edim}(\varphi) .
$$

Definition 2.5. A regular factorization $R \stackrel{\dot{\varphi}}{\rightarrow} R^{\prime} \stackrel{\varphi^{\prime}}{\rightarrow} S$ of a local ring homomorphism $\varphi: R \rightarrow S$ is minimal if $\operatorname{dim}\left(R^{\prime}\right)-\operatorname{dim}(R)=\operatorname{edim}(\varphi)$.

Fact 2.6. Let $\varphi:(R, \mathfrak{m}) \rightarrow(S, \mathfrak{n})$ and $\psi: S \rightarrow T$ be local ring homomorphisms.

(a) By [9, (1.1) Theorem and (1.5) Proposition], if $S$ is complete, then $\varphi$ has a minimal Cohen factorization. Since $\widehat{S}$ is complete, it follows that the semicompletion $\grave{\varphi}: R \rightarrow \widehat{S}$ has a minimal Cohen factorization.

(b) If $\varphi$ is surjective, $\psi$ is weakly regular, and $T$ is complete, then in any minimal Cohen factorization $R \rightarrow R^{\prime} \rightarrow T$ of the composition $\psi \varphi$, we have $T \cong R^{\prime} \otimes_{R} S$, that is, the diagram

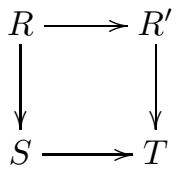

is a pushout; see the proof of [9, (1.6) Theorem].

(c) Assume that $S$ is complete, and consider two Cohen factorizations $R \rightarrow R^{\prime} \rightarrow S$ and $R \rightarrow R^{\prime \prime} \rightarrow S$ of $\varphi$. If the extension $R / \mathfrak{m} \rightarrow S / \mathfrak{n}$ is separable, then there is a comparison $v: R^{\prime} \rightarrow R^{\prime \prime}$ of the first factorization to the second one; moreover, if both Cohen factorizations are minimal, then any comparison between them is an isomorphism. See [9, (1.7) Proposition].

\section{Homological Notions.}

Definition 2.7. The flat dimension of a local ring homomorphism $\varphi: R \rightarrow S$ is $\mathrm{fd}(\varphi)=\mathrm{fd}_{R}(S)$.

The remaining aspects of this subsection use the derived category, so we specify some notations. References on the subject include [14, 20, 30, 31. 
Definition 2.8. The derived category of the category of $R$-modules is denoted $\mathcal{D}(R)$. The objects of this category are the $R$-complexes which we index homologically: $X=\cdots \rightarrow X_{i} \rightarrow X_{i-1} \rightarrow \cdots$.

Let $X$ and $Y$ be $R$-complexes. We say that $X$ is homologically bounded if $\mathrm{H}_{i}(X)=0$ for $|i| \gg 0$, and $X$ is homologically finite if the total homology module $\mathrm{H}(X)=\oplus_{i \in \mathbb{Z}} \mathrm{H}_{i}(X)$ is finitely generated over $R$. The supremum, infimum, and amplitude of $X$ are

$$
\begin{aligned}
\sup (X) & =\sup \left\{i \in \mathbb{Z} \mid \mathrm{H}_{i}(X) \neq 0\right\} \\
\inf (X) & =\inf \left\{i \in \mathbb{Z} \mid \mathrm{H}_{i}(X) \neq 0\right\} \\
\operatorname{amp}(X) & =\sup (X)-\inf (X) .
\end{aligned}
$$

The derived functors of Hom and tensor product are denoted $\mathbf{R H o m}(-,-)$ and $-\otimes_{R}^{\mathbf{L}}-$. For each $i \in \mathbb{Z}$, set $\operatorname{Ext}_{R}^{i}(X, Y):=\mathrm{H}_{-i}\left(\mathbf{R} \operatorname{Hom}_{R}(X, Y)\right)$.

The next definition originates with work of Auslander and Bridger [1, 2].

Definition 2.9. Let $X$ be a homologically finite $R$-complex. Then $X$ is derived reflexive if $\mathbf{R H o m}_{R}(X, R)$ is homologically finite (that is, homologically bounded) and the natural biduality map $X \rightarrow \mathbf{R} \operatorname{Hom}_{R}\left(\mathbf{R H o m}_{R}(X, R), R\right)$ is an isomorphism in $\mathcal{D}(R)$. The $G$-dimension of $X$ is

$$
\mathrm{G}-\operatorname{dim}_{R}(X):= \begin{cases}-\inf \left(\mathbf{R} \operatorname{Hom}_{R}(X, R)\right) & \text { if } X \text { is derived reflexive over } R \\ \infty & \text { otherwise. }\end{cases}
$$

Remark 2.10. For a finitely generated $R$-module, the G-dimension defined above is the same as the one defined by Auslander and Bridger; see [32, 2.7. Theorem]. More generally, the G-dimension of a homologically finite $R$-complex has a similar interpretation by Christensen [11, (2.3.8) GD Corollary].

Given a finitely generated $R$-module $M$, the fact that $\operatorname{Ext}_{R}^{i}(M, R)=0$ for all $i>\mathrm{G}-\operatorname{dim}_{R}(M)$ implies that $\operatorname{grade}_{R}(M) \leqslant \mathrm{G}-\operatorname{dim}_{R}(M)$. This motivates the next definition.

Definition 2.11. A finitely generated $R$-module $M$ is $G$-perfect if $\operatorname{grade}_{R}(M)=$ G-dim $\operatorname{dim}_{R}(M)$.

We proceed with CI-dimension of Avramov, Gasharov, and Peeva [10, 27, 28. and Gerko's CM-dimension [15].

Definition 2.12. Consider a diagram $R \stackrel{\varphi}{\rightarrow} R^{\prime} \stackrel{\tau}{\leftarrow} Q$ of local ring homomorphisms such that $\varphi$ is flat, and $\tau$ is surjective. Such a diagram is a G-quasi-deformation if $R^{\prime}$ is G-perfect as a $Q$-module. Such a diagram is a quasi-deformation if $\operatorname{Ker}(\tau)$ is generated by a $Q$-regular sequence.

Definition 2.13. Let $X$ be a homologically finite complex over a local ring $R$. The $C M$-dimension and CI-dimension of $X$ are

$$
\begin{aligned}
& \mathrm{CM}-\operatorname{dim}_{R}(X):=\inf \left\{\begin{array}{l|l}
\mathrm{G}-\operatorname{dim}_{Q}\left(R^{\prime} \otimes_{R}^{\mathrm{L}} X\right)-\mathrm{G}-\operatorname{dim}_{Q}\left(R^{\prime}\right) & \begin{array}{c}
R \rightarrow R^{\prime} \leftarrow Q \text { is a } \\
\text { G-quasi-deformation }
\end{array}
\end{array}\right\} \\
& \text { CI- } \operatorname{dim}_{R}(X):=\inf \left\{\operatorname{pd}_{Q}\left(R^{\prime} \otimes_{R}^{\mathrm{L}} X\right)-\operatorname{pd}_{Q}\left(R^{\prime}\right) \mid \begin{array}{l}
R \rightarrow R^{\prime} \leftarrow Q \text { is a } \\
\text { quasi-deformation }
\end{array}\right\} .
\end{aligned}
$$


Fact 2.14. Let $\alpha^{\prime}:\left(R^{\prime}, \mathfrak{m}^{\prime}, k^{\prime}\right) \rightarrow\left(\widetilde{R}^{\prime}, \widetilde{\mathfrak{m}}^{\prime}, \widetilde{k}^{\prime}\right)$ be a flat local ring homomorphism, and let $M$ be a homologically finite $R^{\prime}$-complex. The proof of [10, (1.11) Proposition] shows that CI- $\operatorname{dim}_{\widetilde{R}^{\prime}}\left(\widetilde{R}^{\prime} \otimes \mathbb{R}_{R^{\prime}}^{\mathbf{L}} M\right) \geqslant$ CI- $\operatorname{dim}_{R^{\prime}}(M)$ with equality holding when CI-dim $\widetilde{R}_{\widetilde{R}^{\prime}}\left(\widetilde{R}^{\prime} \otimes_{R^{\prime}}^{\mathbf{L}} M\right)<\infty$. A similar argument shows that CM-dim ${\widetilde{R^{\prime}}}\left(\widetilde{R}^{\prime} \otimes_{R^{\prime}}^{\mathbf{L}} M\right) \geqslant$ CM-dim $R_{R^{\prime}}(M)$ with equality holding when CM-dim ${\widetilde{R^{\prime}}}\left(\widetilde{R}^{\prime} \otimes_{R^{\prime}} \mathbf{L}\right)<\infty$.

Definition 2.15. Assume that $(R, \mathfrak{m}, k)$ is local, and let $X$ be a homologically finite $R$-complex. For $i \in \mathbb{Z}$, the $i$ th Bass number and Betti number of $X$ are $\mu_{R}^{i}(X)=\operatorname{rank}_{k}\left(\operatorname{Ext}_{R}^{i}(k, X)\right)$ and $\beta_{i}^{R}(X)=\operatorname{rank}_{k}\left(\operatorname{Ext}_{R}^{i}(X, k)\right)$. The Bass series and Poincaré series of $X$ are the formal Laurent series $I_{R}^{X}(t)=\sum_{i \in \mathbb{Z}} \mu_{R}^{i}(X) t^{i}$ and $P_{X}^{R}(t)=\sum_{i \in \mathbb{Z}} \beta_{i}^{R}(X) t^{i}$.

Definition 2.16. A homologically finite $R$-complex $C$ is semidualizing for $R$ if the natural morphism $R \rightarrow \mathbf{R H o m}_{R}(C, C)$ is an isomorphism in $\mathcal{D}(R)$. A dualizing complex for $R$ is a semidualizing complex $D$ of finite injective dimension, i.e., such that $D$ is isomorphic in $\mathcal{D}(R)$ to a bounded complex of injective $R$-modules.

Remark 2.17. Assume that $R$ is local. Then $R$ has a dualizing complex if and only if it is a homomorphic image of a Gorenstein local ring; one implication is from Grothendieck and Hartshorne [20, and the other is by Kawasaki [23]. In particular, a complete local ring has a dualizing complex.

\section{Properties of Ring Homomorphisms.}

The first notion in this subsection is from Avramov [3].

Definition 2.18. Let $\varphi: R \rightarrow S$ be a local ring homomorphism. Given a Cohen factorization $R \stackrel{\dot{\varphi}}{\rightarrow} R^{\prime} \stackrel{\varphi^{\prime}}{\rightarrow} \widehat{S}$ of the semicompletion $\grave{\varphi}: R \rightarrow \widehat{S}$, we say that $\varphi$ is complete intersection if $\operatorname{Ker}\left(\varphi^{\prime}\right)$ is generated by an $R^{\prime}$-regular sequence.

Remark 2.19. Let $\varphi: R \rightarrow S$ be a local ring homomorphism. The complete intersection property for $\varphi$ is independent of the choice of Cohen factorization by [3, (3.3) Remark]. Also $R$ and $\varphi$ are complete intersection if and only if $S$ is complete intersection and $\mathrm{fd}(\varphi)$ is finite; see [3, (5.9), (5.10), and (5.12)].

The next notion is mostly due to Avramov and Foxby [7, with some contributions from Iyengar and Sather-Wagstaff [22].

Definition 2.20. Let $\varphi: R \rightarrow S$ be a local ring homomorphism. Given a Cohen factorization $R \stackrel{\dot{\varphi}}{\rightarrow} R^{\prime} \stackrel{\varphi^{\prime}}{\rightarrow} \widehat{S}$ of the semicompletion $\grave{\varphi}: R \rightarrow \widehat{S}$, we set

$$
\mathrm{G}-\operatorname{dim}(\varphi):=\mathrm{G}_{-} \operatorname{dim}_{R^{\prime}}(\widehat{S})-\operatorname{edim}(\dot{\varphi}) \text {. }
$$

Remark 2.21. The G-dimension of a local homomorphism is independent of the choice of Cohen factorization by [22, 3.2. Theorem].

Definition 2.22. Let $\varphi: R \rightarrow S$ be a local ring homomorphism, and let $D^{\widehat{R}}$ be a dualizing complex for $\widehat{R}$. A dualizing complex for $\varphi$ is a semidualizing $S$-complex $D^{\varphi}$ such that $D^{\widehat{R}} \otimes \frac{\mathbf{L}}{\widehat{R}}\left(\widehat{S} \otimes_{S}^{\mathbf{L}} D^{\varphi}\right)$ is a dualizing complex for $\widehat{S}$.

Fact 2.23. Let $\varphi: R \rightarrow S$ be a local ring homomorphism of finite G-dimension, e.g., finite flat dimension; see [7, (4.4.2)]. If $S$ is complete, then $\varphi$ has a dualizing complex by [7, (6.7) Lemma]; specifically, given a Cohen factorization $R \rightarrow R^{\prime} \rightarrow S$ of $\varphi$, the $S$-complex $\mathbf{R H o m}_{R^{\prime}}\left(S, R^{\prime}\right)$ is dualizing for $\varphi$. In particular, the semicompletion $\grave{\varphi}: R \rightarrow \widehat{S}$ has a dualizing complex. 
Next are some notions of Avramov and Foxby [7, 8] and Frankild [13.

Definition 2.24. Let $\varphi: R \rightarrow S$ be a local ring homomorphism of finite Gdimension and let $D^{\grave{\varphi}}$ be a dualizing complex for the semicompletion $\grave{\varphi}: R \rightarrow \widehat{S}$. The quasi-Cohen-Macaulay defect of $\varphi$ is $\mathbf{q c m d}(\varphi):=\operatorname{amp}\left(D^{\grave{\varphi}}\right)$, and $\varphi$ is quasiCohen-Macaulay if $\mathbf{q c m d}(\varphi)=0$, that is, if $D^{\grave{\varphi}}$ is isomorphic in $\mathcal{D}(\widehat{S})$ to a module.

When $\operatorname{fd}(\varphi)<\infty$, the Cohen-Macaulay defect of $\varphi$ is $\operatorname{cmd}(\varphi)=\mathbf{q} \operatorname{cmd}(\varphi)$; see [7, (5.5)]. The map $\varphi$ is Cohen-Macaulay if it is quasi-Cohen-Macaulay and has finite flat dimension.

Remark 2.25. Let $\varphi: R \rightarrow S$ be a local ring homomorphism of finite G-dimension. The quasi-Cohen-Macaulay defect of $\varphi$ is independent of the choice of dualizing complex, as the dualizing complex is unique up to shift-isomorphism; see [7, (5.4)] and [13, (6.5)]. If $R$ is Cohen-Macaulay and $\varphi$ is quasi-Cohen-Macaulay, then $S$ is Cohen-Macaulay; the converse holds when $\varphi$ has finite flat dimension or the induced $\operatorname{map} \operatorname{Spec}(\widehat{S}) \rightarrow \operatorname{Spec}(\widehat{R})$ is surjective by [9, (3.10) Theorem] and [13, (7.7)].

Definition 2.26. Let $\varphi: R \rightarrow S$ be a local ring homomorphism of finite Gdimension, and let $D^{\grave{\varphi}}$ be a dualizing complex for the semicompletion $\grave{\varphi}: R \rightarrow \widehat{S}$ such that $\inf \left(D^{\grave{\varphi}}\right)=\operatorname{depth}(S)-\operatorname{depth}(R)$. The Bass series for $\varphi$ is the Poincaré series $I_{\varphi}(t):=P_{D \dot{\varphi}}^{\widehat{S}}(t)$.

Remark 2.27. Let $\varphi: R \rightarrow S$ be a local ring homomorphism of finite G-dimension. The Bass series for $\varphi$ is a formal Laurent series $I_{\varphi}(t)$ with non-negative integer coefficients satisfying the formal relation $I_{S}^{S}(t)=I_{R}^{R}(t) I_{\varphi}(t)$; see [7, (7.1) Theorem].

Definition 2.28. Let $\varphi: R \rightarrow S$ be a local ring homomorphism of finite Gdimension. Then $\varphi$ is quasi-Gorenstein if $I_{\varphi}(t)=t^{\operatorname{depth}(S)-\operatorname{depth}(R)}$, that is, if $\widehat{S}$ is dualizing for $\grave{\varphi}: R \rightarrow \widehat{S}$. The map $\varphi$ is Gorenstein if it is quasi-Gorenstein and has finite flat dimension.

Remark 2.29. If $\varphi: R \rightarrow S$ is a local homomorphism with G-dim $(\varphi)<\infty$, then $S$ is Gorenstein if and only if $R$ is Gorenstein and $\varphi$ is quasi-Gorenstein by [7, (7.7.2)].

The CI-dimension of $\varphi$ is from [28], and the CM-dimension of $\varphi$ works similarly.

Definition 2.30. Let $\varphi: R \rightarrow S$ be a local ring homomorphism. The $C M$ dimension of $\varphi$ and CI-dimension of $\varphi$ are

$$
\begin{aligned}
& \operatorname{CM}-\operatorname{dim}(\varphi):=\inf \left\{\begin{array}{l|l}
\mathrm{CM}-\operatorname{dim}_{R^{\prime}}(\widehat{S})-\operatorname{edim}(\dot{\varphi}) \mid \begin{array}{c}
R \stackrel{\dot{\varphi}}{\rightarrow} R^{\prime} \stackrel{\varphi^{\prime}}{\rightarrow} \widehat{S} \text { is a Cohen } \\
\text { factorization of } \dot{\varphi}
\end{array}
\end{array}\right\} \\
& \text { CI-dim }(\varphi):=\inf \left\{\begin{array}{l|l}
\text { CI- } \operatorname{dim}_{R^{\prime}}(\widehat{S})-\operatorname{edim}(\dot{\varphi}) \mid \begin{array}{c}
R \stackrel{\dot{\varphi}}{\rightarrow} R^{\prime} \stackrel{\varphi^{\prime}}{\rightarrow} \widehat{S} \text { is a Cohen } \\
\text { factorization of } \dot{\varphi}
\end{array}
\end{array}\right\} .
\end{aligned}
$$

Remark 2.31. We do not know whether the finiteness of CM-dimension and/or CI-dimension of a local homomorphism is independent of the choice of Cohen factorization.

\section{Weak Functoriality of Regular/Cohen Factorizations}

The main objective of this section is the proof of Theorem A from the introduction. We begin with a lemma. 
Lemma 3.1. Let $\varphi:(R, \mathfrak{m}) \rightarrow(S, \mathfrak{n})$ be a weakly regular local ring homomorphism. Then $\operatorname{edim}(R)+\operatorname{edim}(S / \mathfrak{m} S)=\operatorname{edim}(S)$.

Proof. Set $e=\operatorname{edim}(R)$, and let $\mathbf{x}=x_{1}, \ldots, x_{e} \in \mathfrak{m}$ be a minimal generating sequence for $\mathfrak{m}$. Set $d=\operatorname{edim}(S / \mathfrak{m} S)$, and let $\mathbf{y}=y_{1}, \ldots, y_{d} \in \mathfrak{n}$ be such that the residue sequence $\overline{\mathbf{y}} \in \mathfrak{n} / \mathfrak{m} S$ generates $\mathfrak{n} / \mathfrak{m} S$ minimally over $S$ and over $S / \mathfrak{m} S$. It is straightforward to show that the concatenated sequence $\varphi(\mathbf{x}), \mathbf{y} \in \mathfrak{n}$ generates $\mathfrak{n}$. It remains to show that this sequence generates $\mathfrak{n}$ minimally.

Suppose by way of contradiction that the sequence $\varphi(\mathbf{x}), \mathbf{y}$ does not generate $\mathfrak{n}$ minimally. The sequence $\varphi(\mathbf{x}), \mathbf{y}$ contains a minimal generating sequence for $\mathfrak{n}$. Thus, either one of the $\varphi\left(x_{i}\right)$ or one of the $y_{j}$ is redundant as part of this generating sequence.

Case 1: $y_{j}$ is redundant as part of this generating sequence. Assume without loss of generality that $j=1$. In this case we have $\mathfrak{n}=\left(\varphi\left(x_{1}\right), \ldots, \varphi\left(x_{e}\right), y_{2}, \ldots, y_{d}\right) S$. Since $\mathfrak{m} S=(\varphi(\mathbf{x})) S$ it follows that the ideal $\mathfrak{n} / \mathfrak{m} S$ is generated by the residue sequence $\overline{y_{2}}, \ldots, \overline{y_{d}}$. This contradicts the minimality of the original sequence $\mathbf{y}$.

Case 2: $\varphi\left(x_{i}\right)$ is redundant as part of generating sequence for $\mathfrak{n}$. Assume without loss of generality that $i=1$. It follows that the ideal $\mathfrak{n} /(\mathbf{y}) S \subset S /(\mathbf{y}) S$ is generated by the residue sequence $\overline{\varphi\left(x_{2}\right)}, \ldots, \overline{\varphi\left(x_{e}\right)} \in \mathfrak{n} /(\mathbf{y}) S$.

The map $\varphi$ is weakly regular, and the sequence $\overline{\mathbf{y}}$ minimally generates the maximal ideal of the regular local ring $S / \mathfrak{m} S$. In particular, this sequence is $S / \mathfrak{m} S$-regular, so we know that the sequence y is $S$-regular and the induced map $R \rightarrow S /(\mathbf{y}) S$ is flat; see, e.g. [24, Corollary to Theorem 22.5]. Since this map is flat and local, it follows from [21, (2.3) Lemma] that the ideal $\mathfrak{m}(S /(\mathbf{y}) S)=\mathfrak{n} /(\mathbf{y}) S$ is minimally generated by the sequence $\overline{\varphi\left(x_{1}\right)}, \overline{\varphi\left(x_{2}\right)}, \ldots, \overline{\varphi\left(x_{e}\right)}$. This contradicts the conclusion of the previous paragraph.

Our next result will provide a vertical map in the proof of Theorem $\mathrm{A}$.

Proposition 3.2. Consider a commutative diagram of local ring homomorphisms

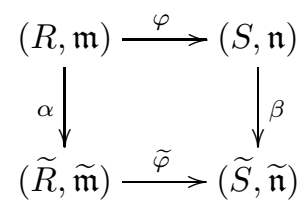

such that $\alpha$ is weakly regular, $\widetilde{S}$ is complete, $\beta$ is weakly regular, and the induced map $R / \mathfrak{m} \rightarrow \widetilde{S} / \widetilde{\mathfrak{n}}$ is separable. Assume that $\varphi$ has a minimal regular factorization $R \stackrel{\dot{\varphi}}{\rightarrow} R^{\prime} \stackrel{\varphi^{\prime}}{\rightarrow} S$, and fix a minimal Cohen factorization $\widetilde{R} \stackrel{\dot{\varphi}}{\rightarrow} S^{\prime} \stackrel{\widetilde{\varphi}^{\prime}}{\rightarrow} \widetilde{S}$ of $\widetilde{\varphi}$. Then there is a weakly regular local ring homomorphism $\alpha^{\prime}: R^{\prime} \rightarrow S^{\prime}$ such that the next diagram commutes

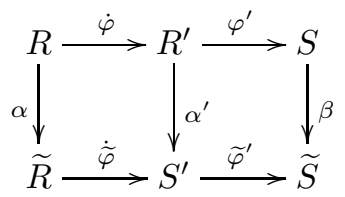

and such that the second square is a pushout. 
Proof. Fact 2.6(b) provides a commutative diagram of local ring homomorphisms

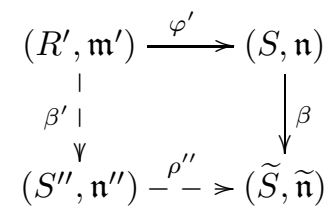

where $R^{\prime} \stackrel{\beta^{\prime}}{\longrightarrow} S^{\prime \prime} \stackrel{\rho^{\prime \prime}}{\longrightarrow} \widetilde{S}$ is a minimal Cohen factorization for $\beta \varphi^{\prime}$ and the diagram is a pushout. Fact 2.2 implies that the diagrams $R \stackrel{\dot{\varphi} \alpha}{\longrightarrow} S^{\prime} \stackrel{\widetilde{\varphi}^{\prime}}{\longrightarrow} \widetilde{S}$ and $R \stackrel{\beta^{\prime} \dot{\varphi}}{\longrightarrow} S^{\prime \prime} \stackrel{\rho^{\prime \prime}}{\longrightarrow} \widetilde{S}$ are Cohen factorizations of $\beta \varphi$. In particular, the next diagram commutes:

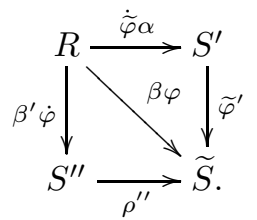

By assumption, the extension $R / \mathfrak{m} \rightarrow \widetilde{S} / \widetilde{\mathfrak{n}}$ is separable. Hence by Fact 2.6(C), there exists a comparison $v: S^{\prime \prime} \rightarrow S^{\prime}$ from $R \stackrel{\beta^{\prime} \dot{\varphi}}{\longrightarrow} S^{\prime \prime} \stackrel{\rho^{\prime \prime}}{\longrightarrow} \widetilde{S}$ to $R \stackrel{\dot{\varphi} \alpha}{\longrightarrow} S^{\prime} \stackrel{\widetilde{\varphi}^{\prime}}{\longrightarrow} \widetilde{S}$. In particular, the following diagram commutes:

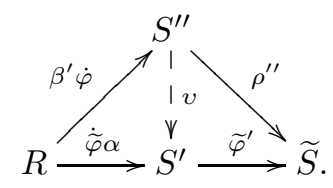

Combining this with (3.2.2), we obtain the next commutative diagram:

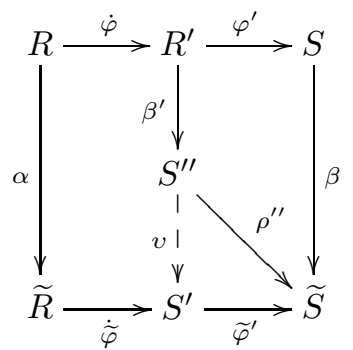

We claim that $v$ is an isomorphism. Once this is shown, it follows from the commutativity of (3.2.3) that the map $\alpha^{\prime}=v \beta^{\prime}$ makes the diagram (3.2.1) commute. The fact that the diagram (3.2.2) is a pushout then implies that the second square in the diagram (3.2.1) is also a pushout. Lastly, the fact that $\beta^{\prime}$ is weakly regular implies that $\alpha^{\prime}$ is also weakly regular, so the desired conclusions hold.

To prove that $v$ is an isomorphism it is enough to show that both Cohen factorizations $R \stackrel{\beta^{\prime} \dot{\varphi}}{\longrightarrow} S^{\prime \prime} \stackrel{\rho^{\prime \prime}}{\longrightarrow} \widetilde{S}$ and $R \stackrel{\dot{\varphi} \alpha}{\longrightarrow} S^{\prime} \stackrel{\widetilde{\varphi}^{\prime}}{\longrightarrow} \widetilde{S}$ are minimal; see Fact 2.6(C). Therefore by definition we need to show the following equalities:

$$
\operatorname{dim}\left(S^{\prime \prime}\right)-\operatorname{dim}(R) \stackrel{(*)}{=} \operatorname{edim}(\widetilde{S} / \mathfrak{m} \widetilde{S}) \stackrel{(\dagger)}{=} \operatorname{dim}\left(S^{\prime}\right)-\operatorname{dim}(R) .
$$


We begin with equality $(*)$. The fact that $R^{\prime} \stackrel{\beta^{\prime}}{\longrightarrow} S^{\prime \prime} \stackrel{\rho^{\prime \prime}}{\longrightarrow} \widetilde{S}$ is a minimal Cohen factorization explains the first step in the next sequence:

$$
\operatorname{dim}\left(S^{\prime \prime}\right)-\operatorname{dim}\left(R^{\prime}\right)=\operatorname{edim}\left(\widetilde{S} / \mathfrak{m}^{\prime} \widetilde{S}\right)=\operatorname{edim}(\widetilde{S} / \mathfrak{n} \widetilde{S})
$$

The second equality is from the fact that the map $R^{\prime} \stackrel{\varphi^{\prime}}{\rightarrow} S$ is surjective. Therefore, since $R \stackrel{\dot{\varphi}}{\rightarrow} R^{\prime} \stackrel{\varphi^{\prime}}{\rightarrow} S$ is a minimal regular factorization, we conclude that

$$
\begin{aligned}
\operatorname{dim}\left(S^{\prime \prime}\right)-\operatorname{dim}(R) & =\left(\operatorname{dim}\left(S^{\prime \prime}\right)-\operatorname{dim}\left(R^{\prime}\right)\right)+\left(\operatorname{dim}\left(R^{\prime}\right)-\operatorname{dim}(R)\right) \\
& =\operatorname{edim}(\widetilde{S} / \mathfrak{n} \widetilde{S})+\operatorname{edim}(S / \mathfrak{m} S)
\end{aligned}
$$

Now, the induced map $\bar{\beta}: S / \mathfrak{m} S \rightarrow \widetilde{S} / \mathfrak{m} \widetilde{S}$ is a flat local homomorphism. Furthermore, the closed fiber of this map is the same as the closed fiber for $\beta$, hence it is regular. Using Lemma 3.1, we conclude that

$$
\operatorname{edim}(\widetilde{S} / \mathfrak{m} \widetilde{S})=\operatorname{edim}(\widetilde{S} / \mathfrak{n} \widetilde{S})+\operatorname{edim}(S / \mathfrak{m} S)
$$

The equality $(*)$ follows from this with the previous display.

Next, we explain the equality $(\dagger)$. By assumption $\widetilde{R} \stackrel{\dot{\varphi}}{\rightarrow} S^{\prime} \stackrel{\widetilde{\varphi}^{\prime}}{\rightarrow} \widetilde{S}$ is a minimal Cohen factorization and $\alpha: R \rightarrow \widetilde{R}$ is a flat local homomorphism. This explains the second step in the next display:

$$
\begin{aligned}
\operatorname{dim}\left(S^{\prime}\right)-\operatorname{dim}(R) & =\left(\operatorname{dim}\left(S^{\prime}\right)-\operatorname{dim}(\widetilde{R})\right)+(\operatorname{dim}(\widetilde{R})-\operatorname{dim}(R)) \\
& =\operatorname{edim}(\widetilde{S} / \widetilde{\mathfrak{m}} \widetilde{S})+\operatorname{dim}(\widetilde{R} / \mathfrak{m} \widetilde{R}) \\
& =\operatorname{edim}(\widetilde{S} / \widetilde{\mathfrak{m}} \widetilde{S})+\operatorname{edim}(\widetilde{R} / \mathfrak{m} \widetilde{R}) \\
& =\operatorname{edim}(\widetilde{S} / \mathfrak{m} \widetilde{S}) .
\end{aligned}
$$

The third step comes from the fact that $\alpha$ is weakly regular. The fourth step is from Fact 2.2 since the maps $R \stackrel{\alpha}{\rightarrow} \widetilde{R} \stackrel{\widetilde{\varphi}}{\rightarrow} \widetilde{S}$ are weakly regular. This display explains $(\dagger)$, and the proof is complete.

Our next result complements the previous one by providing a horizontal map in the proof of Theorem $\mathrm{A}$.

Proposition 3.3. Consider a commutative diagram of local ring homomorphisms

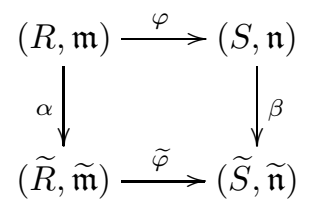


such that $\alpha$ has a regular factorization $R \stackrel{\dot{\alpha}}{\rightarrow} R^{\prime \prime} \stackrel{\alpha^{\prime}}{\rightarrow} \widetilde{R}$, the ring $\widetilde{S}$ is complete, and the field extension $R / \mathfrak{m} \rightarrow \widetilde{S} / \widetilde{\mathfrak{n}}$ is separable. Let $S \stackrel{\dot{\beta}}{\rightarrow} S^{\prime} \stackrel{\beta^{\prime}}{\rightarrow} \widetilde{S}$ be a Cohen factorization of $\beta$. Then there is a commutative diagram of local ring homomorphisms

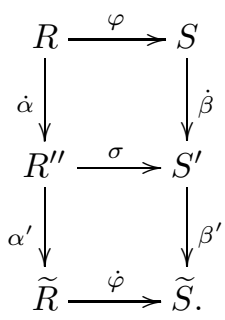

Proof. We prove the result in two cases.

Case 1: the ring $S$ is complete. Let $R \stackrel{\dot{\varphi}}{\rightarrow} R^{\prime} \stackrel{\varphi^{\prime}}{\rightarrow} S$ and $\widetilde{R} \stackrel{\dot{\varphi}}{\rightarrow} \widetilde{R}^{\prime} \stackrel{\widetilde{\varphi}^{\prime}}{\rightarrow} \widetilde{S}$ be Cohen factorizations of $\varphi$ and $\widetilde{\varphi}$, respectively. Since $\widetilde{R}^{\prime}$ and $S^{\prime}$ are complete, the maps $R^{\prime \prime} \stackrel{\dot{\varphi} \alpha^{\prime}}{\longrightarrow} \widetilde{R}^{\prime}$ and $R^{\prime} \stackrel{\dot{\beta} \varphi^{\prime}}{\longrightarrow} S^{\prime}$ have Cohen factorizations $R^{\prime \prime} \stackrel{\dot{\pi}}{\rightarrow} T \stackrel{\pi^{\prime}}{\longrightarrow} \widetilde{R}^{\prime}$ and $R^{\prime} \stackrel{\dot{\tau}}{\rightarrow} T^{\prime} \stackrel{\tau^{\prime}}{\rightarrow} S^{\prime}$, respectively. Since diagram (3.3.1) commutes, one sees readily that $\widetilde{\varphi}^{\prime} \pi^{\prime} \dot{\pi} \dot{\alpha}=\beta^{\prime} \tau^{\prime} \dot{\tau} \dot{\varphi}$. In other words, the diagrams

$$
R \stackrel{\dot{\pi} \dot{\alpha}}{\longrightarrow} T \stackrel{\widetilde{\varphi}^{\prime} \pi^{\prime}}{\longrightarrow} \widetilde{S} \quad R \stackrel{\dot{\tau} \dot{\varphi}}{\longrightarrow} T^{\prime} \stackrel{\beta^{\prime} \tau^{\prime}}{\longrightarrow} \widetilde{S}
$$

are Cohen factorizations of the same map; see Fact 2.2

By assumption, the induced field extension $R / \mathfrak{m} \rightarrow \widetilde{S} / \tilde{\mathfrak{n}}$ is separable. Thus, from Fact 2.6( (c) we conclude that there is a comparison $v: T \rightarrow T^{\prime}$ between the factorizations (3.3.3). It follows that the next diagram commutes

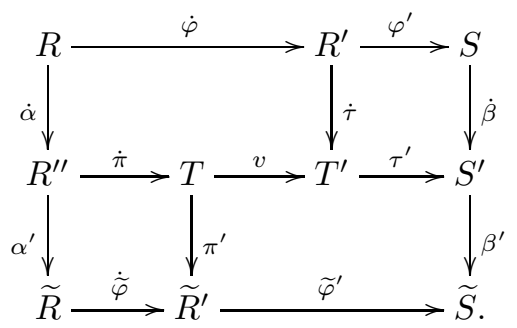

So the map $\sigma=\tau^{\prime} v \dot{\pi}: R^{\prime \prime} \rightarrow S^{\prime}$ makes diagram (3.3.2) commute.

Case 2: the general case. Let $\widehat{(-)}^{\mathfrak{n}}$ denote the $\mathfrak{n}$-adic completion functor, and consider the following commutative diagram of local ring homomorphisms

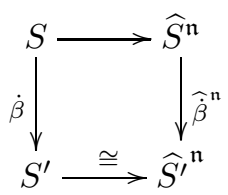

where the horizontal maps are the natural ones. The map $S^{\prime} \rightarrow{\widehat{S^{\prime}}}^{\mathfrak{n}}$ is an isomorphism since $S^{\prime}$ is complete and $\dot{\beta}$ is local. Accordingly, we identify $S^{\prime}$ and $\widehat{S}^{\prime}$, and similarly for $\widetilde{S}$ and $\widehat{\widetilde{S}}^{\mathfrak{n}}$. From [9, (1.9) Remark] we know that the diagram $\widehat{S}^{\mathfrak{n}} \stackrel{\widehat{\hat{\beta}}^{\mathfrak{n}}}{\longrightarrow} S^{\prime} \stackrel{\beta^{\prime}}{\longrightarrow} \widetilde{S}$ is a Cohen factorization of the map $\widehat{S}^{\mathfrak{n}} \stackrel{\widehat{\beta}^{\mathfrak{n}}}{\longrightarrow} \widetilde{S}$. The previous 
diagram provides the second commutative triangle in the next diagram, and the other triangle commutes by definition of $\grave{\varphi}$ :

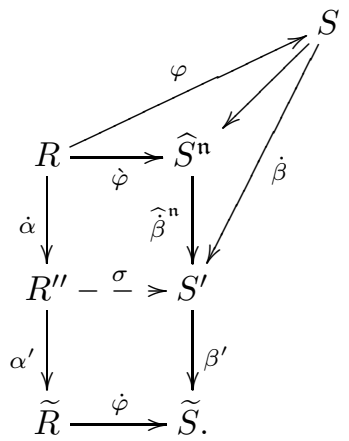

Case 1 provides a local ring homomorphism $\sigma$ making the two squares commute. It follows readily that $\sigma$ also makes the diagram (3.3.2) commute.

3.4 (Proof of Theorem A) . By Proposition 3.3 there is a local ring homomorphism $\sigma: R^{\prime \prime} \rightarrow S^{\prime}$ making the next diagram commute:

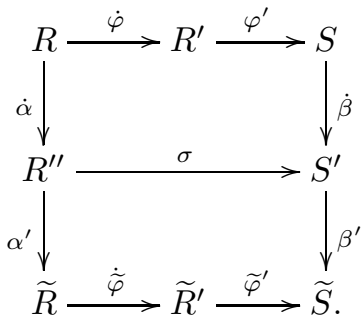

Since $S^{\prime}$ is complete, the map $\sigma$ has a Cohen factorization $R^{\prime \prime} \stackrel{\dot{\sigma}}{\rightarrow} T^{\prime} \stackrel{\sigma^{\prime}}{\rightarrow} S^{\prime}$. Proposition 3.2 provides a weakly regular local ring homomorphism $\delta: R^{\prime} \rightarrow T^{\prime}$ making the next diagram commute, where $R^{\prime \prime} \stackrel{\dot{\pi}}{\rightarrow} T^{\prime \prime} \stackrel{\pi^{\prime}}{\rightarrow} \widetilde{R}^{\prime}$ is a Cohen factorization of $R^{\prime \prime} \stackrel{\dot{\varphi} \alpha^{\prime}}{\longrightarrow} \widetilde{R}^{\prime}$ :

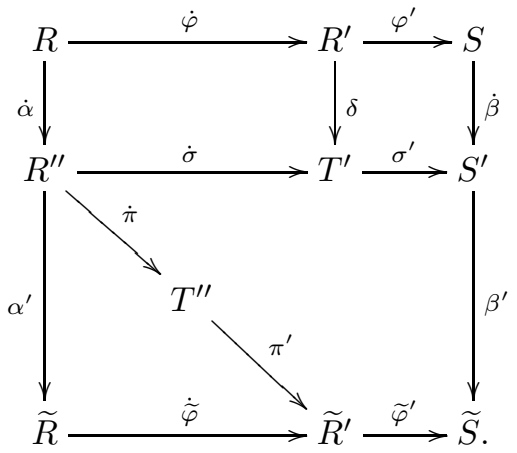

Fact 2.2 implies that the diagrams $R^{\prime \prime} \stackrel{\dot{\pi}}{\rightarrow} T^{\prime \prime} \stackrel{\widetilde{\varphi}^{\prime} \pi^{\prime}}{\longrightarrow} \widetilde{S}$ and $R^{\prime \prime} \stackrel{\dot{\sigma}}{\rightarrow} T^{\prime} \stackrel{\beta^{\prime} \sigma^{\prime}}{\longrightarrow} \widetilde{S}$ are two Cohen factorizations of the map $\widetilde{\varphi} \alpha^{\prime}=\beta^{\prime} \sigma$. By assumption (2), the induced field extension $R / \mathfrak{m} \rightarrow \widetilde{S} / \widetilde{\mathfrak{n}}$ is separable, so there is a comparison $v: T^{\prime} \rightarrow T^{\prime \prime}$ of these Cohen factorizations, by Fact $2.6($ C $)$. This is a local ring homomorphism making 
the next diagram commute:

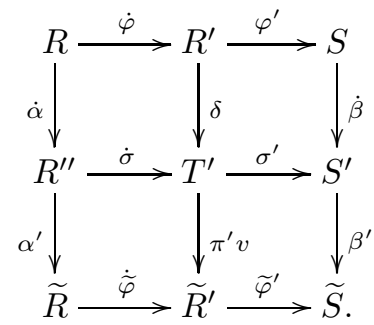

At this point, the only problem is that $\pi^{\prime} v$ may not be surjective. We use a technique from [9, 19, to remedy this.

Since $\widetilde{R}^{\prime}$ and $S^{\prime}$ are local and complete and the maps $\widetilde{\varphi}^{\prime}$ and $\beta^{\prime}$ are surjective, in the pull-back square

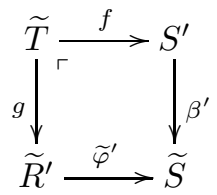

the ring $\widetilde{T}$ is noetherian, local, and complete by [19, (19.3.2.1)]. The commutativity of the diagram (3.4.2) implies that there is a local ring homomorphism $w: T^{\prime} \rightarrow \widetilde{T}$ making the next diagram commute:

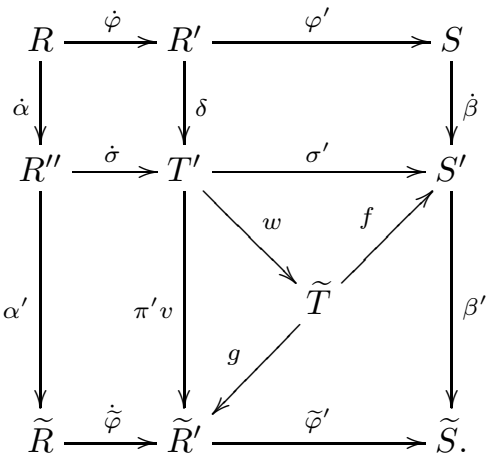

The ring $\widetilde{T}$ is complete, so the map $w$ has a Cohen factorization $T^{\prime} \stackrel{\dot{w}}{\rightarrow} T \stackrel{w^{\prime}}{\rightarrow} \widetilde{T}$. Consider the following diagram

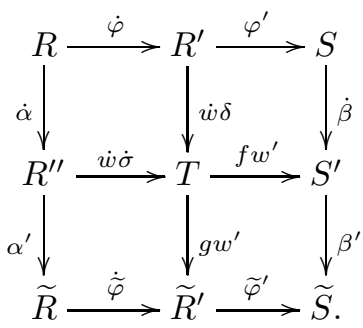

This diagram has the desired properties for (A.2). Indeed, commutativity follows from the commutativity of (3.4.3), using the equation $w=w^{\prime} \dot{w}$, and the middle column and middle row are Cohen factorizations by Fact 2.2 
The following example, from [9, (1.8) Example] shows that the separability assumptions (2) in Theorem $\mathrm{A}$ are necessary.

Example 3.5. In [9, (1.8) Example], the authors construct a local ring homomorphism $\varphi: R \rightarrow S$ with a pair of Cohen factorizations

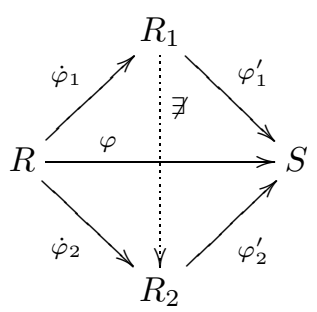

such that there is not a comparison from the top one to the bottom one. (Note that, by Fact 2.6 (c) , the map on residue fields induced by $\varphi$ cannot be separable.) We begin with the following diagram, as in A.1

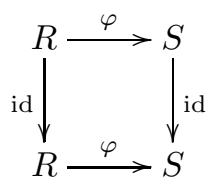

with the given Cohen factorizations represented by the horizontal solid arrows in the next diagram.

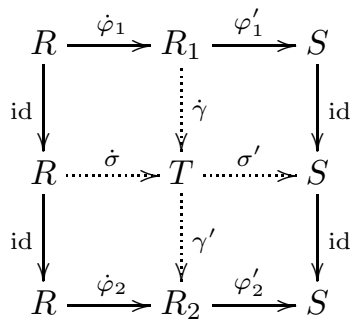

If there were Cohen factorizations (as represented by the dotted arrows in the diagram) then the map $\gamma^{\prime} \dot{\gamma}$ would provide a comparison of the Cohen factorizations from (3.5.1), contradicting the conclusion of [9, (1.8) Example]. Thus, in the notation of Theorem $\mathrm{A}$ the field extension $\widetilde{R} / \widetilde{\mathfrak{m}} \rightarrow \widetilde{S} / \widetilde{\mathfrak{n}}$ must be separable (even when the extension $R / \mathfrak{m} \rightarrow \widetilde{R} / \widetilde{\mathfrak{m}}$ is trivial). Similarly, the reflected diagrams
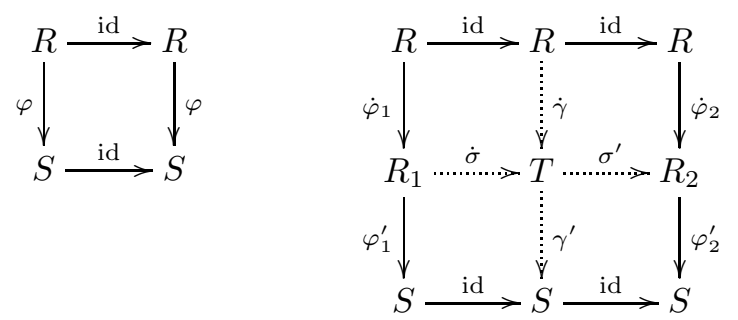

show that the field extension $R / \mathfrak{m} \rightarrow \widetilde{R} / \widetilde{\mathfrak{m}}$ must be separable (even when the extension $\widetilde{R} / \widetilde{\mathfrak{m}} \rightarrow \widetilde{S} / \widetilde{\mathfrak{n}}$ is trivial). 
The next example shows that, even when the factorizations $R \stackrel{\dot{\varphi}}{\rightarrow} R^{\prime} \stackrel{\varphi^{\prime}}{\longrightarrow} S$ and $\widetilde{R} \stackrel{\dot{\varphi}}{\rightarrow} \widetilde{R}^{\prime} \stackrel{\widetilde{\varphi}^{\prime}}{\rightarrow} \widetilde{S}$ are minimal, the map $\gamma=\gamma^{\prime} \dot{\gamma}$ in Theorem $₫$ is not uniquely determined.

Example 3.6. Let $k$ be a field, and consider the following diagram where the unspecified maps are the natural ones:

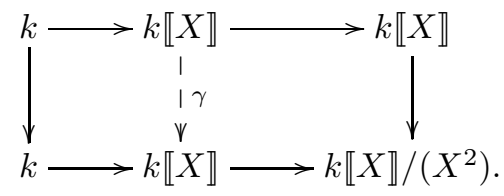

One can define $\gamma$ by mapping $X$ to any power series of the form $X+X^{2} f$ to make the diagram commute.

\section{Properties of Local Ring Homomorphisms in Commuting Diagrams}

In this section, we prove Theorem $\mathrm{B}$ from the introduction.

Lemma 4.1. Fix a commutative diagram of field extensions

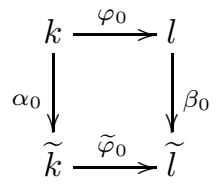

Let $\varphi:(R, \mathfrak{m}, k) \rightarrow(S, \mathfrak{n}, l)$ and $\alpha: R \rightarrow(\widetilde{R}, \widetilde{\mathfrak{m}}, \widetilde{k})$ and $\beta: S \rightarrow(\widetilde{S}, \widetilde{\mathfrak{n}}, \widetilde{l})$ be local ring homomorphisms that induce the maps $\varphi_{0}$ and $\alpha_{0}$ and $\beta_{0}$ on residue fields. If $\alpha$ is weakly Cohen and $\widetilde{S}$ is complete, then there is a commutative diagram of local ring homomorphisms

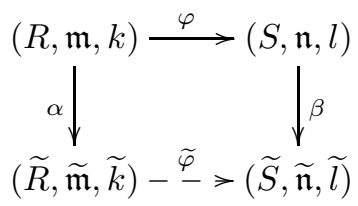

such that $\widetilde{\varphi}$ induces the map $\widetilde{\varphi}_{0}$ on residue fields.

Proof. By assumption, the maps $\alpha$ and $\beta$ make the following diagram commute

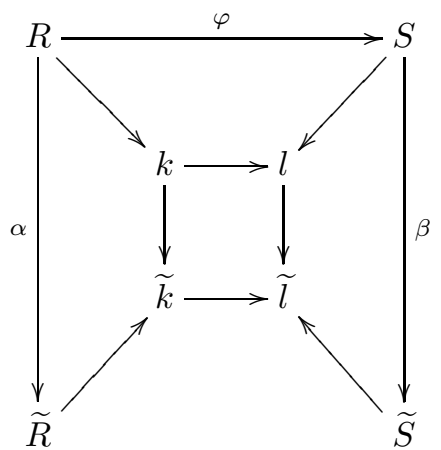


Since the extension $k \hookrightarrow \widetilde{k}$ is separable, the map $\alpha$ is formally smooth by [18, Théorème (19.8.2.i)], so [18, Corollaire (19.3.11)] provides a local homomorphism $\widetilde{\varphi}: \widetilde{R} \rightarrow \widetilde{S}$ making the next diagram commute:

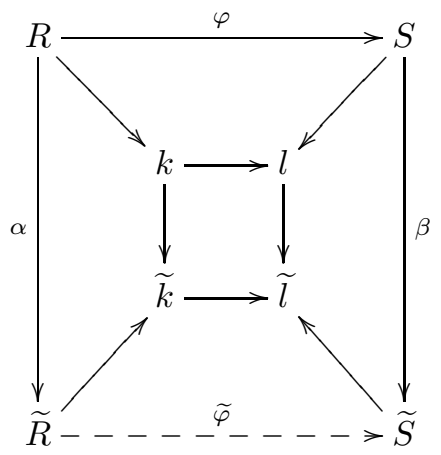

It follows that the outer square in this diagram has the desired properties.

Remark 4.2. Given a diagram (4.1.1), there exist Cohen maps $\alpha$ and $\beta$, as in Lemma 4.1] by [18, Théorèm 19.8.2(ii)].

We use the next result to relate the flat dimensions of the maps in Theorem B

Proposition 4.3. Consider a commutative diagram of local ring homomorphisms

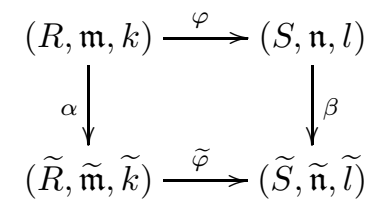

such that $\mathrm{fd}(\alpha)$ and $\mathrm{fd}(\beta)$ are both finite.

(a) One has $\operatorname{fd}(\varphi) \leqslant \mathrm{fd}(\widetilde{\varphi})+\mathrm{fd}(\alpha)$; in particular, if $\operatorname{fd}(\widetilde{\varphi})$ is finite, then so is $\operatorname{fd}(\varphi)$.

(b) If $\alpha$ is weakly regular, then $\operatorname{fd}(\widetilde{\varphi}) \leqslant \operatorname{edim}(\alpha)+\operatorname{fd}(\beta)+\operatorname{fd}(\varphi)$; in particular, $\operatorname{fd}(\varphi)$ and $\operatorname{fd}(\widetilde{\varphi})$ are simultaneously finite.

(c) If $\alpha$ is flat with closed fiber a field and $\beta$ is flat, then $\operatorname{fd}(\widetilde{\varphi})=\operatorname{fd}(\varphi)$.

Proof. (国) This is proved like [22, Theorem 5.7].

(b) Assume without loss of generality that $\operatorname{fd}(\varphi)<\infty$. Let $\mathbf{x}=x_{1}, \ldots, x_{e} \in \widetilde{\mathfrak{m}}$ be a sequence whose residue sequence in $\widetilde{R} / \mathfrak{m} \widetilde{R}$ is a regular system of parameters. This explains the first isomorphism in the derived category $\mathcal{D}(\widetilde{S})$ in the next sequence:

$$
\begin{aligned}
\widetilde{k} \otimes_{\widetilde{R}}^{\mathbf{L}} \widetilde{S} & \simeq\left[(\widetilde{R} / \mathbf{x} \widetilde{R}) \otimes_{\widetilde{R}}^{\mathbf{L}}(\widetilde{R} / \mathfrak{m} \widetilde{R})\right] \otimes_{\widetilde{R}}^{\mathbf{L}} \widetilde{S} \\
& \simeq(\widetilde{R} / \mathbf{x} \widetilde{R}) \otimes_{\widetilde{R}}^{\mathbf{L}}\left[(\widetilde{R} / \mathfrak{m} \widetilde{R}) \otimes_{\widetilde{R}}^{\mathbf{L}} \widetilde{S}\right] \\
& \simeq(\widetilde{R} / \mathbf{x} \widetilde{R}) \otimes_{\widetilde{R}}^{\mathbf{L}}\left[\left(k \otimes_{R}^{\mathbf{L}} \widetilde{R}\right) \otimes_{\widetilde{R}}^{\mathbf{L}} \widetilde{S}\right] \\
& \simeq(\widetilde{R} / \mathbf{x} \widetilde{R}) \otimes_{\widetilde{R}}^{\mathbf{L}}\left[\left(k \otimes_{R}^{\mathbf{L}} S\right) \otimes_{S}^{\mathbf{L}} \widetilde{S}\right] .
\end{aligned}
$$

The second isomorphism is associativity. The third isomorphism is from the flatness of $\alpha$. The fourth isomorphism is from the commutativity of (4.3.1). This explains 
the second step in the next display:

$$
\begin{aligned}
\operatorname{fd}(\widetilde{\varphi}) & =\operatorname{amp}\left(\widetilde{k} \otimes_{\widetilde{R}}^{\mathbf{L}} \widetilde{S}\right) \\
& =\operatorname{amp}\left((\widetilde{R} / \mathbf{x} \widetilde{R}) \otimes_{\widetilde{R}}^{\mathbf{L}}\left[\left(k \otimes_{R}^{\mathbf{L}} S\right) \otimes_{S}^{\mathbf{L}} \widetilde{S}\right]\right) \\
& \leqslant \operatorname{pd}_{\widetilde{R}}(\widetilde{R} / \mathbf{x} \widetilde{R})+\operatorname{amp}\left(\left(k \otimes_{R}^{\mathbf{L}} S\right) \otimes_{S}^{\mathbf{L}} \widetilde{S}\right) \\
& =e+\operatorname{amp}\left(\left(k \otimes_{R}^{\mathbf{L}} S\right) \otimes_{S}^{\mathbf{L}} \widetilde{S}\right) \\
& =e+\operatorname{fd}(\beta \varphi) \\
& \leqslant e+\operatorname{fd}(\beta)+\operatorname{fd}(\varphi) \\
& =\operatorname{edim}(\widetilde{R} / \mathfrak{m} \widetilde{R})+\operatorname{fd}(\beta)+\mathrm{fd}(\varphi) .
\end{aligned}
$$

The first and fifth steps are from [5, Proposition 5.5]. The third step is standard; see [12, (7.28) and (8.17)]. The fourth step is from [24, Corollary to Theorem 22.5], which tells us that $\mathrm{x}$ is $\widetilde{R}$-regular. The sixth step is by [6, (1.8(a))]. The seventh step is by definition of $e$ as $\operatorname{edim}(\widetilde{R} / \mathfrak{m} \widetilde{R})$.

(ㄷ) Part (国) implies that $\mathrm{fd}(\widetilde{\varphi}) \geqslant \mathrm{fd}(\varphi)$, and (b) implies that $\mathrm{fd}(\widetilde{\varphi}) \leqslant \mathrm{fd}(\varphi)$.

Here are some examples that show the limitations of the previous result.

Example 4.4. Let $k$ be a field, and consider the following commutative diagram of natural local ring homomorphisms:

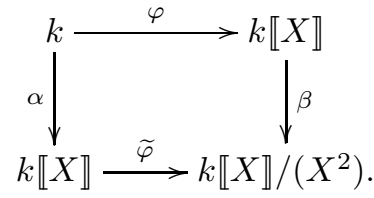

Note that $\alpha$ is weakly regular, and we have

$$
\mathrm{fd}(\widetilde{\varphi})=1<2=1+1+0=\operatorname{edim}(\alpha)+\mathrm{fd}(\beta)+\mathrm{fd}(\varphi)
$$

so we can have strict inequality in Proposition 4.3(b).

Example 4.5. Let $k$ be a field, and let $S$ be a complete local ring with coefficient field $k$, and assume that $S$ is not a field. Consider the following commutative diagram of natural local ring homomorphisms:

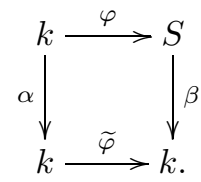

Then we have $\mathrm{fd}(\widetilde{\varphi})=0<0+\mathrm{fd}(\beta)=\mathrm{fd}(\varphi)+\mathrm{fd}(\beta)$. so we can have strict inequality in Proposition 4.3(C) if $\beta$ is not flat.

Lemma 4.6. Let $\left(\widetilde{R}^{\prime}, \widetilde{\mathfrak{m}}^{\prime}, \widetilde{k}^{\prime}\right) \stackrel{\alpha^{\prime}}{\longleftarrow}\left(R^{\prime}, \mathfrak{m}^{\prime}, k^{\prime}\right) \stackrel{\rho}{\rightarrow}\left(R_{1}, \mathfrak{m}_{1}, k_{1}\right) \stackrel{\tau}{\leftarrow}\left(Q, \mathfrak{r}, k_{1}\right)$ be local ring homomorphisms such that $\alpha^{\prime}$ is Cohen, $\rho$ is flat, and $\tau$ is surjective. Fix a commutative diagram

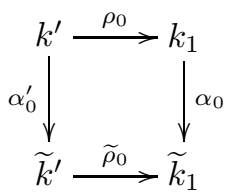


of field extensions such that $\rho_{0}$ and $\alpha_{0}^{\prime}$ are the maps induced on residue fields by $\rho$ and $\alpha^{\prime}$. Fix a weakly regular homomorphism $\alpha_{1}: R_{1} \rightarrow\left(\widetilde{R}_{1}, \widetilde{\mathfrak{m}}_{1}, \widetilde{k}_{1}\right)$ that induces the map $\alpha_{0}$ on residue fields. If $\widetilde{R}_{1}$ is complete, then there is a commutative diagram of local ring homomorphisms

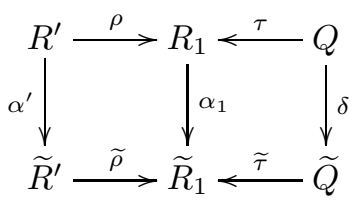

such that $\widetilde{\rho}$ is flat, $\delta$ is weakly regular, the right-hand square is a pushout, and $\widetilde{\rho}$ induces the map $\widetilde{\rho}_{0}$ on residue fields.

Proof. Lemma 4.1 provides a local ring homomorphism $\widetilde{\rho}: \widetilde{R}^{\prime} \rightarrow \widetilde{R}_{1}$ making the left-hand square commute. Proposition 4.3 (c) implies that $\tilde{\rho}$ is flat. We get the right-hand square from Fact 2.6(b).

The next result augments [10, (1.13) Proposition].

Proposition 4.7. Let $\alpha^{\prime}:\left(R^{\prime}, \mathfrak{m}^{\prime}, k^{\prime}\right) \rightarrow\left(\widetilde{R}^{\prime}, \widetilde{\mathfrak{m}}^{\prime}, \widetilde{k}^{\prime}\right)$ be a Cohen homomorphism, and let $M$ be a homologically finite $R^{\prime}$-complex. Then we have

(a) CI-dim ${\widetilde{R^{\prime}}}\left(\widetilde{R}^{\prime} \otimes_{R^{\prime}}^{\mathbf{L}} M\right)=\mathrm{CI}-\operatorname{dim}_{R^{\prime}}(M)$; thus, the quantities CI-dim ${\widetilde{R^{\prime}}}_{\widetilde{R}^{\prime}} \otimes_{R^{\prime}}^{\mathbf{L}} M)$ and CI-dim $R_{R^{\prime}}(M)$ are simultaneously finite.

(b) CM-dim $\widetilde{R}_{\widetilde{R}^{\prime}}\left(\widetilde{R}^{\prime} \otimes_{R^{\prime}}^{\mathbf{L}} M\right)=\mathrm{CM}-\operatorname{dim}_{R^{\prime}}(M)$; in particular, CM-dim ${\widetilde{R^{\prime}}}_{\widetilde{R}^{\prime}} \otimes_{R^{\prime}}^{\mathbf{L}} M)$ and $\mathrm{CM}-\operatorname{dim}_{R^{\prime}}(M)$ are simultaneously finite.

Proof. (国) It suffices by Fact 2.14 to assume that CI-dim $R_{R^{\prime}}(M)$ is finite and prove that CI- $\operatorname{dim}_{\widetilde{R}^{\prime}}\left(\widetilde{R}^{\prime} \otimes \widetilde{R}_{\widetilde{R}^{\prime}}^{\mathbf{L}} M\right)$ is finite as well. Let $R^{\prime} \stackrel{\rho}{\rightarrow}\left(R_{1}, \mathfrak{m}_{1}, k_{1}\right) \stackrel{\tau}{\leftarrow}\left(Q, \mathfrak{r}, k_{1}\right)$ be a quasi-deformation such that $\operatorname{pd}_{Q}\left(R_{1} \otimes_{R^{\prime}}^{\mathbf{L}} M\right)<\infty$. Let $\widetilde{k}_{1}$ be a join of $k_{1}$ and $\widetilde{k}^{\prime}$ over $k^{\prime}$, so there is a commutative diagram

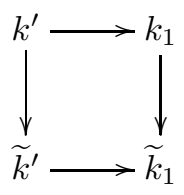

of field extensions. There is a weakly regular homomorphism $\alpha_{1}: R_{1} \rightarrow\left(\widetilde{R}_{1}, \widetilde{\mathfrak{m}}_{1}, \widetilde{k}_{1}\right)$ that induces the map $k_{1} \rightarrow \widetilde{k}_{1}$ on residue fields by [17, Proposition (0.10.3.1)]. Compose with the natural map from $\widetilde{R}_{1}$ to its completion if necessary to assume that $\widetilde{R}_{1}$ is complete. Thus, Lemma 4.6 provides a commutative diagram of local ring homomorphisms

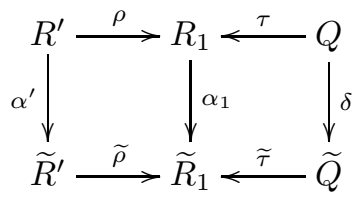

such that $\widetilde{\rho}$ is flat, $\delta$ is weakly regular, and the right-hand square is a pushout. In particular, the bottom row of this diagram is a quasi-deformation. Also, in the 
following sequence, the second and fourth equalities are from the flatness of $\delta$ :

$$
\begin{aligned}
\operatorname{pd}_{\widetilde{Q}}\left(\widetilde{R}_{1} \otimes_{\widetilde{R}^{\prime}}^{\mathbf{L}}\left(\widetilde{R}^{\prime} \otimes_{R^{\prime}}^{\mathbf{L}} M\right)\right) & =\operatorname{pd}_{\widetilde{Q}}\left(\widetilde{R}_{1} \otimes_{R^{\prime}}^{\mathbf{L}} M\right) \\
& =\operatorname{pd}_{\widetilde{Q}}\left(\left(\widetilde{Q} \otimes_{Q}^{\mathbf{L}} R_{1}\right) \otimes_{R^{\prime}}^{\mathbf{L}} M\right) \\
& =\operatorname{pd}_{\widetilde{Q}}\left(\widetilde{Q} \otimes_{Q}^{\mathbf{L}}\left(R_{1} \otimes_{R^{\prime}}^{\mathbf{L}} M\right)\right) \\
& =\operatorname{pd}_{Q}\left(R_{1} \otimes_{R^{\prime}}^{\mathbf{L}} M\right) .
\end{aligned}
$$

By definition, it follows that CI- $\operatorname{dim}_{\widetilde{R}^{\prime}}\left(\widetilde{R}^{\prime} \otimes \widetilde{R}^{\prime}, M\right)$ is finite, as desired.

(b) This is proved as above, starting with a G-quasi-deformation $\left(R^{\prime}, \mathfrak{m}^{\prime}, k^{\prime}\right) \stackrel{\rho}{\rightarrow}$ $\left(R_{1}, \mathfrak{m}_{1}, k_{1}\right) \stackrel{\tau}{\leftarrow}\left(Q, \mathfrak{r}, k_{1}\right)$, and observing that the diagram $\widetilde{R}^{\prime} \stackrel{\widetilde{\rho}}{\rightarrow} \widetilde{R}_{1} \stackrel{\widetilde{\tau}}{\leftarrow} \widetilde{Q}$ is also a a G-quasi-deformation. This follows from the fact that the flat base change of a G-perfect ideal is G-perfect, which is readily checked.

The next result compares to Proposition 4.3, It is important for our proof of Theorem $\mathrm{B}$ from the introduction.

Proposition 4.8. Consider a commutative diagram of local ring homomorphisms

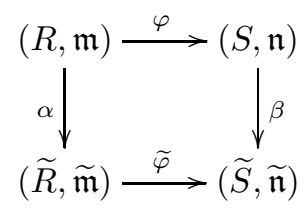

such that $\alpha$ is weakly Cohen, $\beta$ is weakly regular, and the induced map $\widetilde{R} / \widetilde{\mathfrak{m}} \rightarrow \widetilde{S} / \widetilde{\mathfrak{n}}$ is separable. Then we have

(a) $\mathrm{G}-\operatorname{dim}(\widetilde{\varphi})=\mathrm{G}-\operatorname{dim}(\varphi)+\operatorname{edim}(\alpha)-\operatorname{edim}(\beta)$. Hence, the quantities $\mathrm{G}-\operatorname{dim}(\varphi)$ and $\mathrm{G}-\operatorname{dim}(\widetilde{\varphi})$ are simultaneously finite.

(b) If $\beta$ is Cohen, then CI- $\operatorname{dim}(\widetilde{\varphi})=\mathrm{CI}-\operatorname{dim}(\varphi)+\operatorname{edim}(\alpha)$, so the quantities $\mathrm{CI}-\operatorname{dim}(\varphi)$ and $\mathrm{CI}-\operatorname{dim}(\widetilde{\varphi})$ are simultaneously finite.

(c) If $\beta$ is Cohen, then $\mathrm{CM}-\operatorname{dim}(\widetilde{\varphi})=\mathrm{CM}-\operatorname{dim}(\varphi)+\operatorname{edim}(\alpha)$, so the quantities $\mathrm{CM}-\operatorname{dim}(\varphi)$ and $\mathrm{CM}-\operatorname{dim}(\widetilde{\varphi})$ are simultaneously finite.

Proof. (b) Assume that $\beta$ is Cohen.

Case 1: $S$ and $\widetilde{S}$ are complete. Let $R \stackrel{\dot{\varphi}}{\rightarrow} R^{\prime} \stackrel{\varphi^{\prime}}{\longrightarrow} S$ be a minimal Cohen factorization of $\varphi$, and let $\widetilde{R} \stackrel{\dot{\varphi}}{\rightarrow} S^{\prime} \stackrel{\widetilde{\varphi}^{\prime}}{\rightarrow} \widetilde{S}$ be a minimal Cohen factorization of $\widetilde{\varphi}$. Proposition 3.2 provides a weakly regular local ring homomorphism $\alpha^{\prime}: R^{\prime} \rightarrow S^{\prime}$ such that the next diagram commutes

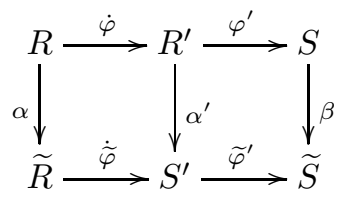

and such that the second square is a pushout. The residue field extension induced by $\alpha^{\prime}$ is the same as the one induced by $\beta$, since the maps $\varphi^{\prime}$ and $\widetilde{\varphi}^{\prime}$ are surjective. Thus, the fact that $\alpha^{\prime}$ is weakly regular and $\beta$ is Cohen implies that $\alpha^{\prime}$ is weakly Cohen. Furthermore, $\alpha^{\prime}$ and $\beta$ have isomorphic closed fibers, so $\alpha^{\prime}$ is Cohen. 
The pushout condition and the flatness of $\alpha^{\prime}$ explain the first equality in the next display

$$
\text { CI-dim } S_{S^{\prime}}(\widetilde{S})=\text { CI-dim } S_{S^{\prime}}\left(S^{\prime} \otimes_{R^{\prime}}^{\mathbf{L}} S\right)=\text { CI-dim } \operatorname{Rim}_{R^{\prime}}(S)
$$

and the second equality is from Proposition 4.7(国). With Fact 2.2 this explains the second equality in the next display:

$$
\begin{aligned}
\mathrm{CI}-\operatorname{dim}(\widetilde{\varphi}) & =\mathrm{CI}-\operatorname{dim}_{S^{\prime}}(\widetilde{S})-\operatorname{edim}(\dot{\widetilde{\varphi}}) \\
& =\mathrm{CI}-\operatorname{dim}_{R^{\prime}}(S)-\left[\operatorname{edim}(\dot{\varphi})+\operatorname{edim}\left(\alpha^{\prime}\right)-\operatorname{edim}(\alpha)\right] \\
& =\left[\mathrm{CI}-\operatorname{dim} R_{R^{\prime}}(S)-\operatorname{edim}(\dot{\varphi})\right]-\operatorname{edim}(\beta)+\operatorname{edim}(\alpha) \\
& =\mathrm{CI}-\operatorname{dim}(\varphi)+\operatorname{edim}(\alpha) .
\end{aligned}
$$

The first and last equalities are by definition, since $\beta$ is Cohen; the third equality follows from the fact that $\alpha^{\prime}$ and $\beta$ have isomorphic closed fibers.

Case 2: the general case. There is a natural ring homomorphism $\widehat{\beta}: \widehat{S} \rightarrow \widehat{\widetilde{S}}$ making the following diagram commute

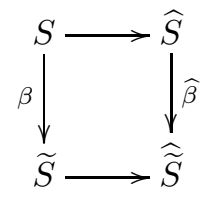

where the horizontal maps are the natural ones. Proposition 4.3 (c) implies that $\widehat{\beta}$ is flat, and it is straightforward to show that its closed fiber is isomorphic to the completion of the closed fiber of $\beta$. Thus, $\widehat{\beta}$ is Cohen. With [28, 2.14.2] this explains the first and third equalities in the next display:

$$
\text { CI- } \operatorname{dim}(\widetilde{\varphi})=\text { CI-dim }(\grave{\varphi})=\text { CI-dim }(\grave{\varphi})+\operatorname{edim}(\alpha)=\text { CI-dim }(\varphi)+\operatorname{edim}(\alpha) .
$$

The second equality is from Case 1 applied to the diagram

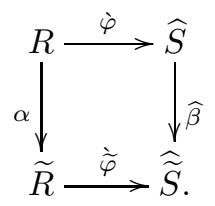

This completes the proof of part (b).

For parts (国) and (ㄷ), argue as above, using [7, (4.1.4)] and Proposition 4.7(b) in place of Proposition 4.7(a) .

4.9 (Proof of Theorem $\mathrm{B}$ ). Case 1: $\mathrm{P}=$ Gorenstein. Since $\alpha$ and $\beta$ are both flat with Gorenstein closed fibers, they are both Gorenstein. It follows that there are integers $a$ and $b$ such that $I_{\widetilde{R}}^{\widetilde{R}}(t)=t^{a} I_{R}^{R}(t)$ and $I_{\widetilde{S}}^{\widetilde{S}}(t)=t^{b} I_{S}^{S}(t)$. Thus, there is an integer $c$ such that $I_{S}^{S}(t)=t^{c} I_{R}^{R}(t)$ if and only if there is an integer $d$ such that $I_{\widetilde{S}}(t)=t^{d} I_{\widetilde{R}}^{\widetilde{R}}(t)$. Proposition $4.3(\mathrm{~b})$ says that $\operatorname{fd}(\widetilde{\varphi})$ is finite if and only if $\operatorname{fd}(\varphi)$ is finite, so $\varphi$ is Gorenstein if and only if $\widetilde{\varphi}$ is Gorenstein, by definition.

Case 2: $\mathrm{P}=$ quasi-Gorenstein. This follows like Case 1, using Proposition 4.8(国).

For the remaining cases, let $R \stackrel{\dot{\varphi}}{\rightarrow} R^{\prime} \stackrel{\varphi^{\prime}}{\longrightarrow} S$ be a minimal Cohen factorization of $\grave{\varphi}$, and let $\widetilde{R} \stackrel{\dot{\varphi}}{\rightarrow} S^{\prime} \stackrel{\widetilde{\varphi}^{\prime}}{\longrightarrow} \widetilde{S}$ be a minimal Cohen factorization of $\dot{\varphi}$. Proposition 3.2 
provides a weakly regular local ring homomorphism $\alpha^{\prime}: R^{\prime} \rightarrow S^{\prime}$ such that the next diagram commutes

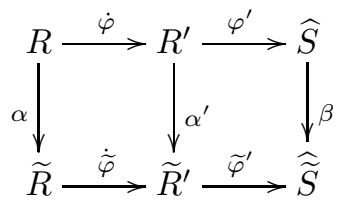

and such that the second square is a pushout.

Case 3: $\mathrm{P}=$ complete intersection. Since the second square of diagram (4.9.1) is a pushout, we have $\operatorname{Ker}\left(\widetilde{\varphi}^{\prime}\right)=\operatorname{Ker}\left(\varphi^{\prime}\right) \widetilde{R}^{\prime}$. The fact that $\alpha^{\prime}$ is flat and local implies that a minimal generating sequence for $\operatorname{Ker}\left(\varphi^{\prime}\right)$ extends to a minimal generating sequence for $\operatorname{Ker}\left(\varphi^{\prime}\right) \widetilde{R}^{\prime}$, and that this sequence is $R^{\prime}$-regular if and only if it is $\widetilde{R}^{\prime}$ regular. That is, the ideal $\operatorname{Ker}\left(\widetilde{\varphi}^{\prime}\right)=\operatorname{Ker}\left(\varphi^{\prime}\right) \widetilde{R}^{\prime}$ in $\widetilde{R}^{\prime}$ is a complete intersection if and only if $\operatorname{Ker}\left(\varphi^{\prime}\right) \subseteq R^{\prime}$ is a complete intersection. Thus, $\varphi$ is complete intersection if and only if $\widetilde{\varphi}$ is complete intersection, by definition.

Case 4: $\mathrm{P}=$ (quasi-)Cohen-Macaulay. Assume for the moment that G-dim $(\varphi)<$ $\infty$. Then Proposition 4.8(a) implies that G- $\operatorname{dim}(\widetilde{\varphi})<\infty$. From [7, (6.7) Lemma], a relative dualizing complex for $\grave{\varphi}$ is $D^{\grave{\varphi}}=\mathbf{R H o m}_{R^{\prime}}\left(\widehat{S}, R^{\prime}\right)$, and it follows that

$$
\begin{aligned}
D^{\grave{\varphi}} & =\mathbf{R H o m}_{\widetilde{R}^{\prime}}\left(\widehat{\widetilde{S}}, \widetilde{R}^{\prime}\right) \\
& \simeq \mathbf{R H o m}_{\widetilde{R}^{\prime}}\left(\widetilde{R}^{\prime} \otimes_{R^{\prime}}^{\mathbf{L}} \widehat{S}, \widetilde{R}^{\prime} \otimes_{R^{\prime}}^{\mathbf{L}} R^{\prime}\right) \\
& \simeq \widetilde{R}^{\prime} \otimes_{R^{\prime}}^{\mathbf{L}} \mathbf{R H o m}_{R^{\prime}}\left(\widehat{S}, R^{\prime}\right) \\
& =\widetilde{R}^{\prime} \otimes_{R^{\prime}}^{\mathbf{L}} D^{\grave{\varphi}} .
\end{aligned}
$$

The second step is from the fact that the second square of diagram (4.9.1) is a pushout and $\alpha^{\prime}$ is flat. It follows that

$$
\begin{aligned}
\operatorname{qcmd}(\widetilde{\varphi}) & =\mathbf{q c m d}(\grave{\varphi})=\operatorname{amp}\left(D^{\grave{\varphi}}\right)=\operatorname{amp}\left(\widetilde{R}^{\prime} \otimes_{R^{\prime}}^{\mathbf{L}} D^{\grave{\varphi}}\right) \\
& =\operatorname{amp}\left(D^{\grave{\varphi}}\right)=\mathbf{q c m d}(\grave{\varphi})=\mathbf{q c m d}(\varphi) .
\end{aligned}
$$

A similar argument shows that if $\operatorname{fd}(\varphi)<\infty$, then $\operatorname{cmd}(\widetilde{\varphi})=\operatorname{cmd}(\varphi)$.

In view of the simultaneous finiteness given in Propositions 4.3 (ad $)$ (b) and 4.8 (ad), we see that $\varphi$ is quasi-Cohen-Macaulay if and only if $\widetilde{\varphi}$ is quasi-Cohen-Macaulay, and $\varphi$ is Cohen-Macaulay if and only if $\widetilde{\varphi}$ is Cohen-Macaulay.

Remark 4.10. We have learned from Javier Majadas and Tirdad Sharif that the assumptions of weak Cohenness and separability can be removed from Theorem B in the case where $\mathrm{P}$ is "complete intersection", using André-Quillen homology. In the other cases, though, we do not know if these assumptions are necessary.

\section{Proof of Theorem C}

Remark 5.1. Complete intersection dimension is quite nice in many respects. However, at this time we do not know how it behaves with respect to short exact sequences: If two of the modules in an exact sequence $0 \rightarrow M_{1} \rightarrow M_{2} \rightarrow M_{3} \rightarrow 0$ have finite CI-dimension, must the third module also have finite CI-dimension?

The difficulty with this question is the following. Assume, for instance, that CI- $\operatorname{dim}_{R}\left(M_{1}\right)$ and CI- $\operatorname{dim}_{R}\left(M_{2}\right)$ are finite. Then for $i=1,2$ there is a quasideformation $R \rightarrow R_{i} \leftarrow Q_{i}$ such that $\operatorname{pd}_{Q_{i}}\left(R_{i} \otimes_{R} M_{i}\right)<\infty$. If there were a single 
quasi-deformation $R \rightarrow R^{\prime} \leftarrow Q$ such that $\operatorname{pd}_{Q}\left(R^{\prime} \otimes_{R} M_{i}\right)<\infty$ for $i=1,2$ then we could conclude easily that $\operatorname{pd}_{Q}\left(R^{\prime} \otimes_{R} M_{3}\right)<\infty$, so CI- $\operatorname{dim}_{R}\left(M_{3}\right)<\infty$. The difficulty lies in attempting to combine the two given quasi-deformations into a single one that works for both $M_{1}$ and $M_{2}$. Theorem C deals with half of this problem when the maps $R \rightarrow R_{i}$ are weakly Cohen by showing how to combine the flat maps in the given quasi-deformations. However, we do not see how to use Theorem $\mathrm{C}$ to answer the above question in any special cases, e.g., when $R$ contains a field of characteristic 0 .

5.2 (Proof of Theorem C) . (国) Consider the induced maps $k \rightarrow k_{i}$ for $i=1,2$. Since these are separable by assumption, there is a commutative diagram of separable field extensions

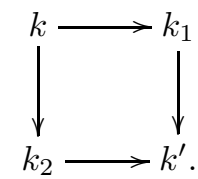

Let $\alpha:(R, \mathfrak{m}, k) \rightarrow\left(\widetilde{R}, \widetilde{\mathfrak{m}}, k^{\prime}\right)$ and $\beta_{i}:\left(R_{i}, \mathfrak{m}_{i}, k_{i}\right) \rightarrow\left(\widetilde{R}_{i}, \widetilde{\mathfrak{m}}_{i}, k^{\prime}\right)$ be Cohen extensions corresponding to the separable field extensions $k \rightarrow k^{\prime}$ and $k_{i} \rightarrow k^{\prime}$ such that the rings $\widetilde{R}$ and $\widetilde{R}_{i}$ are complete; see [18, Théorèm 19.8.2(ii)].

By Lemma 4.1 and Proposition 4.3 (c), each $\varphi_{i}(i=1,2)$ can be extended to a flat local homomorphism $\widetilde{\varphi}_{i}$ making the following diagram commute:

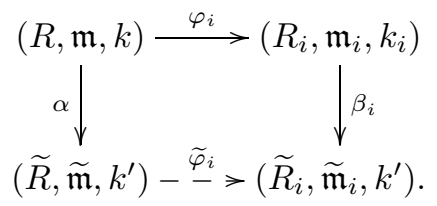

For $i=1,2$, Fact 2.6(b) provides a commutative diagram of local ring homomorphisms

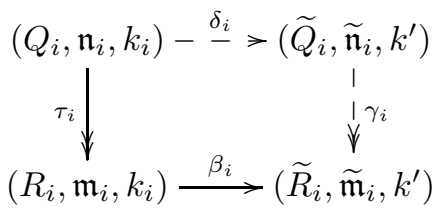

such that $\widetilde{Q}_{i}$ is complete, $\delta_{i}$ is weakly regular, $\gamma_{i}$ is surjective, and the induced map $R_{i} \otimes_{Q_{i}} \widetilde{Q}_{i} \rightarrow \widetilde{R}_{i}$ is an isomorphism. Because of this isomorphism and the flatness of $\delta_{i}$, the fact that $\tau_{i}$ is surjective with kernel generated by a $Q_{i}$-regular sequence implies that $\gamma_{i}$ is surjective with kernel generated by a $\widetilde{Q}_{i}$-regular sequence. Also, since the maps $R \stackrel{\varphi_{i}}{\longrightarrow} R_{i} \stackrel{\beta_{i}}{\longrightarrow} \widetilde{R}_{i}$ are flat and local, the same is true of the composition. Thus, for $i=1,2$, the diagram $R \stackrel{\beta_{i} \varphi_{i}}{\longrightarrow} \widetilde{R}_{i} \stackrel{\gamma_{i}}{\longleftarrow} \widetilde{Q}_{i}$ is a quasideformation.

Now consider the complete tensor product $R^{\prime}=\widetilde{R}_{1} \widehat{\otimes}_{\widetilde{R}} \widetilde{R}_{2}$; see, e.g., [16, Section 0.7.7] and [29, Section V.B.2] for background on this. This is a complete semi-local noetherian ring that is flat over each $\widetilde{R}_{i}$ by [18, Lemme 19.7.1.2]. Moreover, the proof of [18, Lemme 19.7.1.2] shows that the maximal ideals of $R^{\prime}$ are in bijection with the maximal ideals of $\left(\widetilde{R}_{1} / \widetilde{\mathfrak{m}}_{1}\right) \otimes_{\widetilde{R}}\left(\widetilde{R}_{2} / \widetilde{\mathfrak{m}}_{2}\right) \cong k^{\prime} \otimes_{k^{\prime}} k^{\prime} \cong k^{\prime}$, so $R^{\prime}$ is local. For $i=1,2$ let $\sigma_{i}:\left(\widetilde{R}_{i}, \widetilde{\mathfrak{m}}_{i}, k^{\prime}\right) \rightarrow\left(R^{\prime}, \mathfrak{m}^{\prime}, k^{\prime}\right)$ be the natural (flat local) map. 
Hence, each $\sigma_{i}$ is flat with complete target, so by Fact 2.6(2.6) there exists a commutative diagram of local homomorphisms

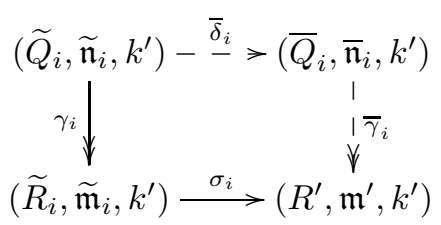

such that $\bar{Q}_{i}$ is complete, $\bar{\delta}_{i}$ is weakly regular, and $\bar{\gamma}_{i}$ is surjective.

Thus, the following diagram

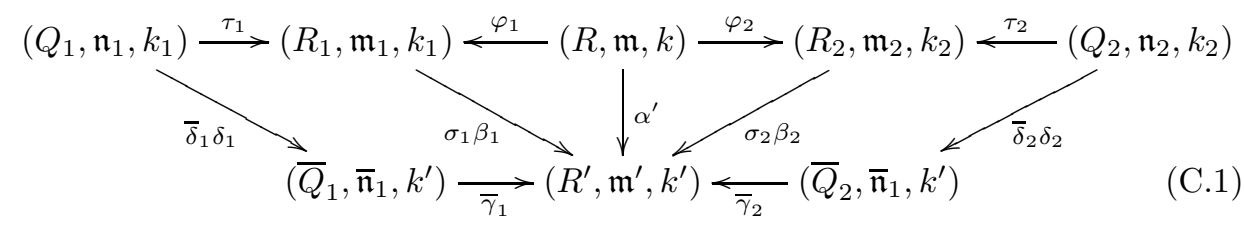

commutes where $\alpha^{\prime}=\sigma_{1} \beta_{1} \varphi_{1}=\sigma_{2} \beta_{2} \varphi_{2}$. By assumption, each $\bar{\gamma}_{i}$ is surjective. Since $\sigma_{1}, \beta_{1}$, and $\varphi_{1}$ are flat, so is their composition $\alpha^{\prime}$. The maps $\bar{\delta}_{i}$ and $\delta_{i}$ are weakly regular, hence Fact 2.2 implies that their composition is weakly regular. Moreover, since the field extension $k_{i} \rightarrow k^{\prime}$ is separable, the composition $\bar{\delta}_{i} \delta_{i}$ is weakly Cohen.

(b) Assume that each map $\varphi_{i}$ is weakly Cohen. Then the closed fiber $R_{i} / \mathfrak{m} R_{i}$ is regular. Since $\beta_{i}: R_{i} \rightarrow \widetilde{R}_{i}$ is Cohen, the same is true of the induced map $R_{i} / \mathfrak{m} R_{i} \rightarrow \widetilde{R}_{i} / \mathfrak{m} \widetilde{R}_{i}$. Thus, the fact that $R_{i} / \mathfrak{m} R_{i}$ is regular implies that $\widetilde{R}_{i} / \mathfrak{m} \widetilde{R}_{i}$ is also regular. Since $\alpha: R \rightarrow \widetilde{R}$ is Cohen, we have $\mathfrak{m} \widetilde{R}=\widetilde{\mathfrak{m}}$, and it follows that $\widetilde{R}_{i} / \mathfrak{m} \widetilde{R}_{i}=\widetilde{R}_{i} / \widetilde{\mathfrak{m}} \widetilde{R}_{i}$ is regular. From [18, Lemme 19.7.1.2] we know that the closed fiber of the map $\sigma_{1}:\left(\widetilde{R}_{1}, \widetilde{\mathfrak{m}}_{1}, k^{\prime}\right) \rightarrow\left(R^{\prime}, \mathfrak{m}^{\prime}, k^{\prime}\right)$ is

$R^{\prime} / \widetilde{\mathfrak{m}}_{1} R^{\prime} \cong\left[\widetilde{R}_{1} / \widetilde{\mathfrak{m}}_{1}\right] \otimes_{\widetilde{R}} \widetilde{R}_{2} \cong\left[\widetilde{R}_{1} / \widetilde{\mathfrak{m}}_{1}\right] \otimes_{k^{\prime}}\left[\widetilde{R}_{2} / \widetilde{\mathfrak{m}} \widetilde{R}_{2}\right] \cong k^{\prime} \otimes_{k^{\prime}}\left[\widetilde{R}_{2} / \widetilde{\mathfrak{m}} \widetilde{R}_{2}\right] \cong \widetilde{R}_{2} / \widetilde{\mathfrak{m}} \widetilde{R}_{2}$

Since this ring is regular and the field extension $k^{\prime} \rightarrow k^{\prime}$ is trivially separable, the map $\sigma_{1}$ is weakly Cohen, as is $\sigma_{2}$ by similar argument. Thus, Fact 2.6 (b) implies that diagram (5.2.2) is a pushout. As in an earlier part of this proof, for each $i=1,2$ the diagram $R \stackrel{\sigma_{i} \beta_{i} \varphi_{i}}{\longrightarrow} R^{\prime} \stackrel{\bar{\gamma}_{i}}{\longleftarrow} \bar{Q}_{i}$ is a quasi-deformation.

By construction, the maps $R_{i} \stackrel{\sigma_{i} \beta_{i}}{\longrightarrow} R^{\prime}$ are compositions of weakly Cohen maps, so they are weakly Cohen; hence conclusion (b1) from the statement of Theorem C is satisfied. Since the diagrams (5.2.1) and (5.2.2) are pushouts, the same is true of the parallelograms in the diagram above; hence conclusion (b2) from the statement of Theorem $\mathrm{C}$ is satisfied. Lastly, given an $R$-module $M$, the flatness of the map $Q_{i} \stackrel{\bar{\delta}_{i} \delta_{i}}{\longrightarrow} \bar{Q}_{i}$ provides the first equality in the next display:

$$
\begin{aligned}
\operatorname{pd}_{Q_{i}}\left(M \otimes_{R} R_{i}\right) & =\operatorname{pd}_{\bar{Q}_{i}}\left(\left(M \otimes_{R} R_{i}\right) \otimes_{Q_{i}} \bar{Q}_{i}\right) \\
& =\operatorname{pd}_{\bar{Q}_{i}}\left(M \otimes_{R}\left(R_{i} \otimes_{Q_{i}} \bar{Q}_{i}\right)\right) \\
& =\operatorname{pd}_{\bar{Q}_{i}}\left(M \otimes_{R} R^{\prime}\right) .
\end{aligned}
$$

(See [25, Theorem 9.6] and [26].) The last equality is from the pushout conclusion on each parallelogram. This shows that conclusion (b3) from the statement of Theorem $\mathrm{C}$ is satisfied. 
Remark 5.3. At this time, we do not know if the weakly Cohen assumption is necessary in Theorem C

\section{ACKNOWLEDGMENTS}

We are grateful to Javier Majadas, Tirdad Sharif, and the referee for their thoughtful and useful comments.

\section{REFERENCES}

1. M. Auslander, Anneaux de Gorenstein, et torsion en algèbre commutative, Séminaire d'Algèbre Commutative dirigé par Pierre Samuel, vol. 1966/67, Secrétariat mathématique, Paris, 1967. MR 37 \#1435

2. M. Auslander and M. Bridger, Stable module theory, Memoirs of the American Mathematical Society, No. 94, American Mathematical Society, Providence, R.I., 1969. MR 42 \#4580

3. L. L. Avramov, Locally complete intersection homomorphisms and a conjecture of Quillen on the vanishing of cotangent homology, Ann. of Math. (2) 150 (1999), no. 2, 455-487. MR 1726700 (2001a:13024)

4. L. L. Avramov and H.-B. Foxby, Gorenstein local homomorphisms, Bull. Amer. Math. Soc. (N.S.) 23 (1990), no. 1, 145-150. MR 1020605 (90k:13009)

5. - Homological dimensions of unbounded complexes, J. Pure Appl. Algebra 71 (1991), 129-155. MR 93g:18017

6. L Locally Gorenstein homomorphisms, Amer. J. Math. 114 (1992), no. 5, 1007-1047. MR 1183530 (93i:13019)

7. 75 (1997), no. 2, 241-270. MR 98d:13014

8. Cohen-Macaulay properties of ring homomorphisms, Adv. Math. 133 (1998), no. 1, 54-95. MR 1492786 (99c:13043)

9. L. L. Avramov, H.-B. Foxby, and B. Herzog, Structure of local homomorphisms, J. Algebra 164 (1994), 124-145. MR 95f:13029

10. L. L. Avramov, V. N. Gasharov, and I. V. Peeva, Complete intersection dimension, Inst. Hautes Études Sci. Publ. Math. (1997), no. 86, 67-114 (1998). MR 1608565 (99c:13033)

11. L. W. Christensen, Gorenstein dimensions, Lecture Notes in Mathematics, vol. 1747, SpringerVerlag, Berlin, 2000. MR 2002e:13032

12. H.-B. Foxby, Hyperhomological algebra 83 commutative rings, lecture notes.

13. A. Frankild, Quasi Cohen-Macaulay properties of local homomorphisms, J. Algebra 235 (2001), 214-242. MR 2001j:13023

14. S. I. Gelfand and Y. I. Manin, Methods of homological algebra, Springer-Verlag, Berlin, 1996. MR 2003m:18001

15. A. A. Gerko, On homological dimensions, Mat. Sb. 192 (2001), no. 8, 79-94, translation in Sb. Math. 192 (2001), no. 7-8, 1165-1179. MR 2002h:13024

16. A. Grothendieck, Éléments de géométrie algébrique. I. Le langage des schémas, Inst. Hautes Études Sci. Publ. Math. (1960), no. 4, 228. MR 0217083 (36 \#177a)

17. A. Grothendieck, Éléments de géométrie algébrique. III. Étude cohomologique des faisceaux cohérents. I, Inst. Hautes Études Sci. Publ. Math. (1961), no. 11, 167. MR 0217085 (36 $\# 177 \mathrm{c})$

18. Éléments de géométrie algébrique. IV. Étude locale des schémas et des morphismes de schémas. I, Inst. Hautes Études Sci. Publ. Math. (1964), no. 20, 259.

19. _ Éléments de géométrie algébrique. IV. Étude locale des schémas et des morphismes de schémas IV, Inst. Hautes Études Sci. Publ. Math. (1967), no. 32, 361.

20. R. Hartshorne, Residues and duality, Lecture Notes in Mathematics, No. 20, Springer-Verlag, Berlin, 1966. MR 36 \#5145

21. B. Herzog, On the macaulayfication of local rings, J. Algebra 67 (1980), no. 2, 305-317. MR 0602065 (82c:13029)

22. S. Iyengar and S. Sather-Wagstaff, G-dimension over local homomorphisms. Applications to the Frobenius endomorphism, Illinois J. Math. 48 (2004), no. 1, 241-272. MR 2048224 (2005c:13016) 
23. T. Kawasaki, On Macaulayfication of Noetherian schemes, Trans. Amer. Math. Soc. 352 (2000), no. 6, 2517-2552. MR 1707481 (2000j:14077)

24. H. Matsumura, Commutative ring theory, second ed., Studies in Advanced Mathematics, vol. 8, University Press, Cambridge, 1989. MR 90i:13001

25. A. Perry, Faithfully flat descent for projectivity of modules, preprint (2012) arXiv: $1011.0038 \mathrm{v} 1$.

26. M. Raynaud and L. Gruson, Critères de platitude et de projectivité. Techniques de "platification" d'un module, Invent. Math. 13 (1971), 1-89. MR 0308104 (46 \#7219)

27. S. Sather-Wagstaff, Complete intersection dimensions for complexes, J. Pure Appl. Algebra 190 (2004), no. 1-3, 267-290. MR 2043332 (2005i:13022)

28. Complete intersection dimensions and Foxby classes, J. Pure Appl. Algebra 212 (2008), no. 12, 2594-2611. MR 2452313 (2009h:13015)

29. J.-P. Serre, Algèbre locale. Multiplicités, Cours au Collège de France, 1957-1958, rédigé par Pierre Gabriel. Seconde édition, 1965. Lecture Notes in Mathematics, vol. 11, Springer-Verlag, Berlin, 1965. MR 0201468 (34 \#1352)

30. J.-L. Verdier, Catégories dérivées, SGA $4 \frac{1}{2}$, Springer-Verlag, Berlin, 1977, Lecture Notes in Mathematics, Vol. 569, pp. 262-311. MR 57 \#3132

31. Des catégories dérivées des catégories abéliennes, Astérisque (1996), no. 239, xii+253 pp. (1997), With a preface by Luc Illusie, Edited and with a note by Georges Maltsiniotis. MR 98c:18007

32. S. Yassemi, G-dimension, Math. Scand. 77 (1995), no. 2, 161-174. MR 97d:13017

Department of Mathematics, North Dakota State University Dept \# 2750, PO Box 6050, FARGO, ND 58108-6050 USA

E-mail address: saeed.nasseh@ndsu.edu

URL: https://www.ndsu.edu/pubweb/ nasseh/

E-mail address: sean.sather-wagstaff@ndsu.edu

$U R L:$ http://www.ndsu.edu/pubweb/ ${ }^{\text {ssatherw/ }}$ 